\title{
Average decay of Fourier transforms and geometry of convex sets
}

\section{Luca Brandolini, Marco Rigoli and Giancarlo Travaglini}

\begin{abstract}
Let $B$ be a convex body in $\mathbb{R}^{2}$, with piecewise smooth boundary and let $\widehat{\chi}_{B}$ denote the Fourier transform of its characteristic function. In this paper we determine the admissible decays of the spherical $L^{p}$-averages of $\widehat{\chi}_{B}$ and we relate our analysis to a problem in the geometry of convex sets. As an application we obtain sharp results on the average number of integer lattice points in large bodies randomly positioned in the plane.
\end{abstract}

\section{Introduction.}

Given a convex body $B$, that is, a compact convex set with non empty interior in $\mathbb{R}^{n}$, we denote by $\chi_{B}$ its characteristic function. The study of the decay of the Fourier transform

$$
\widehat{\chi}_{B}(\xi)=\int_{B} e^{-2 \pi i \xi \cdot x} d x
$$

as $|\xi| \longrightarrow \infty$, in terms of the geometric properties of $B$, is a fascinating and by now classical subject (see [18, Chapter VIII] for basic results, related problems and references). For instance, it is well known that, when the boundary is smooth with everywhere strictly positive Gauss- 
Kronecker curvature, the order of decay of $\widehat{\chi}_{B}$ in a given direction is independent of this latter.

This situation is far from being typical, as one can easily check by considering either a cube or any convex body with a smooth boundary containing flat points. Furthermore, a number of problems requires some sort of "global information" on the decay of $\widehat{\chi}_{B}(\xi)$ which is not a direct consequence of the presently known directional estimates.

In this setting, the study of the spherical $L^{p}$-averages

$$
\left(\int_{\Sigma_{n-1}}\left|\widehat{\chi}_{B}(\rho \sigma)\right|^{p} d \sigma\right)^{1 / p}
$$

turns out to be quite useful.

We point out that the $L^{2}$ case has been investigated by various authors, notably [14], [15], [19], [13], [12]; while for general $p$ 's and $B$ a polyhedron, a detailed analysis with applications to problems on lattice points and on irregularities of distributions can be found in [3]. We note that the $L^{1}$ case is also naturally related with the summability of multiple Fourier integrals (see e.g. [2] or [4] ), moreover, F. Ricci and one of us (G. Travaglini) have recently shown that the general $L^{p}$ case is connected to boundedness of Radon transforms (see [16]).

Throughout this paper, unless otherwise explicitly stated, we consider convex bodies $B$ in $\mathbb{R}^{2}$ with piecewise smooth boundary. More precisely, we assume that $\partial B$ is a union of a finite number of regular arcs, each one of them being $C^{\infty}$ in its interior.

According to a more general result of Podkorytov [13], (see also [19] ) the $L^{2}$-average decay of $\widehat{\chi}_{B}$ satisfies

$$
\left(\int_{0}^{2 \pi}\left|\widehat{\chi}_{B}(\rho \Theta)\right|^{2} d \theta\right)^{1 / 2} \leq c \rho^{-3 / 2},
$$

where, from now on,

$$
\Theta=(\cos \theta, \sin \theta), \quad \theta \in[0,2 \pi),
$$

$\rho \geq 1$ and $c, c_{1}, c_{2}, \ldots$, denote positive constants independent of $\rho$ which may change from line to line.

It is an easy consequence of a result of Montgomery [12, p. 116] that (1.1) is sharp. Namely, for any $B$,

$$
\limsup _{\rho \rightarrow \infty} \rho^{3 / 2}\left(\int_{0}^{2 \pi}\left|\widehat{\chi}_{B}(\rho \Theta)\right|^{2} d \theta\right)^{1 / 2}>0
$$


We stress that in the $L^{2}$ case the order of decay is independent of $B$. The aim of this paper is to study the general $L^{p}$ case where the results turn out to depend on the shape of $B$.

It is worth to begin with the case of a polygon $P$. It has been proved in [3] that

$$
\begin{aligned}
& \left(\int_{0}^{2 \pi}\left|\widehat{\chi}_{P}(\rho \Theta)\right|^{p} d \theta\right)^{1 / p} \\
& \quad \leq \begin{cases}c \rho^{-2} \log (1+\rho), & \text { when } p=1, \\
c \rho^{-1-1 / p}, & \text { when } 1<p \leq \infty .\end{cases}
\end{aligned}
$$

Here we prove

$$
\int_{0}^{2 \pi}\left|\widehat{\chi}_{P}(\rho \Theta)\right| d \theta \geq c \rho^{-2} \log (1+\rho)
$$

and, for each $1<p \leq \infty$

$$
\limsup _{\rho \rightarrow \infty} \rho^{1+1 / p}\left(\int_{0}^{2 \pi}\left|\widehat{\chi}_{P}(\rho \Theta)\right|^{p} d \theta\right)^{1 / p}>0
$$

Next, we consider the case when $B$ is not a polygon. We show that

$$
\limsup _{\rho \rightarrow \infty} \rho^{3 / 2}\left(\int_{0}^{2 \pi}\left|\widehat{\chi}_{B}(\rho \Theta)\right|^{p} d \theta\right)^{1 / p}>0
$$

whenever $1 \leq p \leq \infty$ (note that, when $p=2,(1.4)$ and (1.5) agree with (1.2)). These results, when compared with (1.1) and (1.3), completely describe the case $1 \leq p \leq 2$. As for $p \geq 2$, an easy interpolation argument between $p=2$ and $p=\infty$ gives

$$
\left(\int_{0}^{2 \pi}\left|\widehat{\chi}_{B}(\rho \Theta)\right|^{p} d \theta\right)^{1 / p} \leq c \rho^{-1-1 / p},
$$

for every $2 \leq p \leq \infty$. Contrary to the case $1 \leq p<2$, in the range $2<p \leq \infty$ every order of decay between $\rho^{-3 / 2}$ and $\rho^{-1-1 / p}$ is possible. More precisely we exhibit, for any $2 \leq p \leq \infty$ and $1+1 / p \leq a \leq 3 / 2$, a corresponding convex body $B$ such that

$$
c_{1} \rho^{-a} \leq\left(\int_{0}^{2 \pi}\left|\widehat{\chi}_{B}(\rho \Theta)\right|^{p} d \theta\right)^{1 / p} \leq c_{2} \rho^{-a} .
$$


When $1+1 / p<a<3 / 2$ such examples are constructed so to have, for a suitable $\gamma>2$, a piece of the curve of equation $y=|x|^{\gamma}$ in its boundary. As a side-product, we obtain a result on the average decay of the Fourier transforms of singular measures supported on the above curves (see Proposition 3.17 below).

The different results for $p<2$ and $p>2$ are due to the following fact. When $B$ is not a polygon, its boundary $\partial B$ must contain points with positive curvature and for $1 \leq p \leq 2$ they give the relevant contribution to

$$
\left(\int_{0}^{2 \pi}\left|\widehat{\chi}_{B}(\rho \Theta)\right|^{p} d \theta\right)^{1 / p}
$$

On the other hand, when $2 \leq p \leq \infty$ the main contribution is given by the flat points (if any), as one may guess considering the $L^{\infty}$ case.

We summarize the main results discussed so far in Figure 1. For $p>1$ and $a>0$ the point $(1 / p, a)$ is marked black if and only if there exists $B$ satisfying

$$
\left(\int_{0}^{2 \pi}\left|\widehat{\chi}_{B}(\rho \Theta)\right|^{p} d \theta\right)^{1 / p}<c \rho^{-a}
$$

and

$$
\limsup _{\rho \rightarrow \infty} \rho^{a}\left(\int_{0}^{2 \pi}\left|\widehat{\chi}_{B}(\rho \Theta)\right|^{p} d \theta\right)^{1 / p}>0
$$

Figure 1. 
It is natural to ask whether (1.4) and (1.5) can be turned into estimates from below. As a matter of fact, a negative answer is given by the two simplest examples of convex bodies in $\mathbb{R}^{2}$ : the square (see Lemma 3.12) and the disc (because of the zeroes of the Bessel function $\left.J_{1}\right)$. On the other hand, we show that, for any $1 \leq p \leq \infty$ and for some polygons $P$, we have

$$
\left(\int_{0}^{2 \pi}\left|\widehat{\chi}_{P}(\rho \Theta)\right|^{p} d \theta\right)^{1 / p} \geq c_{2} \rho^{-1-1 / p},
$$

while, if $B$ is neither a polygon nor a body too "similar" (see Definition 3.3) to a disc, then

$$
\int_{0}^{2 \pi}\left|\widehat{\chi}_{B}(\rho \Theta)\right| d \theta \geq c \rho^{-3 / 2} .
$$

The above results are organized in our main theorem of Section 2. We stress that such general $L^{p}$ estimates hold provided $\partial B$ is piecewise smooth. In Section 4 we shall see that in the framework of arbitrary convex bodies one can find very "chaotic" situations.

A basic tool in some of our proofs is the following known fact.

Let $S_{\theta}=\sup _{x \in B} x \cdot \Theta$. For $\delta>0$ sufficiently small we define, see Figure 2, the set

$$
A_{B}(\delta, \theta)=\left\{x \in B: S_{\theta}-\delta \leq x \cdot \Theta \leq S_{\theta}\right\} .
$$


Then (see Lemma 3.8, [5], or [13])

$$
\left|\widehat{\chi}_{B}(\rho \Theta)\right| \leq c\left(\left|A_{B}\left(\rho^{-1}, \theta\right)\right|+\left|A_{B}\left(\rho^{-1}, \theta+\pi\right)\right|\right),
$$

where $|K|$ denotes the Lebesgue measure of a measurable set $K$.

As a consequence, for each $p \geq 1$,

$$
\left(\int_{0}^{2 \pi}\left|\widehat{\chi}_{B}(\rho \Theta)\right|^{p} d \theta\right)^{1 / p} \leq c\left(\int_{0}^{2 \pi}\left|A_{B}\left(\rho^{-1}, \theta\right)\right|^{p} d \theta\right)^{1 / p}
$$

providing a way to estimate the average decay of $\widehat{\chi}_{B}$ from above. Moreover we shall see ( $c f$. also [5]) that (1.7) can be reversed under additional assumptions on $B$.

Observe that the right hand side of (1.7) does not involve any Fourier transform and the problem of estimating

$$
\left(\int_{0}^{2 \pi}\left|A_{B}(\delta, \theta)\right|^{p} d \theta\right)^{1 / p}
$$

as $\delta \longrightarrow 0$, is indeed a genuine problem in the geometry of convex sets. To the best of our knowledge, such a problem has never been considered before and the closest area in the field is perhaps the study of floating bodies (see e.g. [17]). In Section 5 we shall investigate the admissible decays of

$$
\left(\int_{0}^{2 \pi}\left|A_{B}(\delta, \theta)\right|^{p} d \theta\right)^{1 / p}
$$

as $\delta \longrightarrow 0$, mostly as a consequence of the similar problem for $\widehat{\chi}_{B}$.

We end the paper by applying some of the previous results to a problem on the number of lattice points in a large convex planar body $\rho B$.

Elementary geometric considerations show that

$$
\operatorname{card}\left(\rho B \cap \mathbb{Z}^{2}\right) \sim \rho^{2}|B|
$$

and

$$
\operatorname{card}\left(\rho B \cap \mathbb{Z}^{2}\right)-\rho^{2}|B|=O(\rho)
$$

as $\rho \longrightarrow \infty$. The improvement of (1.8) and the related problems constitute a whole area of research (see e.g. [11] or [8]), where the pointwise estimate (1.8) is often substituted by mean estimates. 
Here we consider a large convex body $\rho B$ randomly positioned in the plane. More precisely, for $\sigma \in S O(2)$ and $t \in \mathbb{R}^{2}$ we study the discrepancy

$$
D_{B}(\rho, \sigma, t)=\operatorname{card}\left(\left(\rho \sigma^{-1}(B)-t\right) \cap \mathbb{Z}^{2}\right)-\rho^{2}|B|,
$$

where $\rho \sigma^{-1}(B)-t$ is a rotated, dilated and translated copy of $B$. Since this function is periodic with respect to the variable $t$ we restrict this latter to $\mathbb{T}^{2}=\mathbb{R}^{2} / \mathbb{Z}^{2}$. Kendall ([10]) has proved $L^{2}$ estimates related to the above discrepancy (see also [3]). Here we prove that if $B$ is a convex planar body with piecewise smooth boundary, different from a polygon, then, for any $1 \leq p \leq 2$,

$$
c_{1} \rho^{1 / 2} \leq\left\|D_{B}(\rho, \cdot, \cdot)\right\|_{L^{p}\left(S O(2) \times \mathbb{T}^{2}\right)} \leq c_{2} \rho^{1 / 2} .
$$

We do not know whether (1.9) holds for some $p>2$. We point out that, in general, it is false when $p=\infty$. Indeed, as a consequence of Hardy's $\Omega$-result for the circle problem (see [7] or [11]) we have, for a $\operatorname{disc} D$,

$$
\limsup _{\rho \rightarrow \infty} \rho^{-1 / 2}(\log \rho)^{-1 / 4}\left\|D_{D}(\rho, \cdot, \cdot)\right\|_{L^{\infty}\left(S O(2) \times \mathbb{T}^{2}\right)}>0 .
$$

\section{Statement of the main result.}

Let $\Sigma_{1}$ be the unit circle in $\mathbb{R}^{2}$. For any complex measurable function $g$ on $\Sigma_{1}$ and for any $p \geq 1$, let

$$
\|g\|_{L^{p}\left(\Sigma_{1}\right)}=\left(\int_{0}^{2 \pi}|g(\Theta)|^{p} d \theta\right)^{1 / p},
$$

where $d \theta$ is the normalized Lebesgue measure. As usual we set

$$
\|g\|_{L^{\infty}\left(\Sigma_{1}\right)}=\operatorname{ess~sup}_{\Theta \in \Sigma_{1}}|g(\Theta)|
$$

Let $B$ be a convex body in $\mathbb{R}^{2} ; \varphi:[1,+\infty) \longrightarrow \mathbb{R}^{+}$a non-increasing function and let $1 \leq p \leq \infty$. We say that $\varphi$ is an optimal estimate of the $p$-average decay of $\widehat{\chi}_{B}$ whenever

i) $\left\|\widehat{\chi}_{B}(\rho \cdot)\right\|_{L^{p}\left(\Sigma_{1}\right)} \leq c \varphi(\rho)$, 
ii) $\limsup _{\rho \rightarrow \infty} \frac{\left\|\widehat{\chi}_{B}(\rho \cdot)\right\|_{L^{p}\left(\Sigma_{1}\right)}}{\varphi(\rho)}>0$. vided

Similarly, $\varphi$ is a sharp estimate of the $p$-average decay of $\widehat{\chi}_{B}$ pro-

$$
c_{1} \varphi(\rho) \leq\left\|\widehat{\chi}_{B}(\rho \cdot)\right\|_{L^{p}\left(\Sigma_{1}\right)} \leq c_{2} \varphi(\rho) .
$$

Our main result essentially concerns the case $\varphi(\rho)=\rho^{-a}$ and the following definition will be useful.

Definition 2.1. When $\varphi(\rho)=\rho^{-a}$ is an optimal or sharp estimate of the p-average decay of $\widehat{\chi}_{B}$ we say that the p-average decay of $\widehat{\chi}_{B}$ has optimal order a or sharp order a respectively.

With this preparation we state our main result.

Theorem 2.2. I) Let $1<p \leq \infty$ and define

$$
\begin{gathered}
S=\left\{\left(\frac{1}{p}, a\right): 1<p<2, a=\frac{3}{2} \text { or } a=1+\frac{1}{p}\right\}, \\
T=\left\{\left(\frac{1}{p}, a\right): 2 \leq p \leq \infty, 1+\frac{1}{p} \leq a \leq \frac{3}{2}\right\} .
\end{gathered}
$$

The following are equivalent:

i) There exists a convex body $B$ with piecewise $C^{\infty}$ boundary such that the p-average decay of $\widehat{\chi}_{B}$ has optimal order a.

ii) $(1 / p, a) \in S \cup T$.

II) Let $p=1$. If $P$ is a polygon then $\varphi(\rho)=\rho^{-2} \log (1+\rho)$ is an optimal estimate for the 1-average decay of $\widehat{\chi}_{P}$. If $B$ is any other convex body with piecewise $C^{\infty}$ boundary, then the 1-average decay of $\widehat{\chi}_{B}$ has optimal order $3 / 2$.

Moreover it will be clear from the proof that this result still holds after substituting the word "optimal" with the word "sharp".

The above theorem will be obtained as a consequence of the following somewhat more informative results.

In the first Proposition we cover the case $1 \leq p \leq 2$ when $B$ is not a polygon. 
Proposition 2.3. Let $1 \leq p \leq 2$ and let $B$ be a convex body with piecewise $C^{\infty}$ boundary. Suppose $B$ is not a polygon, then $3 / 2$ is the optimal order of the p-average decay of $\widehat{\chi}_{B}$. Moreover, $3 / 2$ is the sharp order of the p-average decay of $\hat{\chi}_{B}$ for some, but not for all, bodies $B$.

The above Proposition follows from Lemma 3.1, Lemma 3.2, Lemma 3.6 and the example of the disc.

We now consider the case of a polygon.

Proposition 2.4. Let $P$ be a compact convex polygon with non empty interior. Then $\varphi(\rho)=\rho^{-2} \log (1+\rho)$ is a sharp estimate of the 1 average decay of $\hat{\chi}_{P}$. If $1<p \leq \infty$, then $1+1 / p$ is the optimal order of the p-average decay of $\hat{\chi}_{P}$. Moreover, $1+1 / p$ is the sharp order of the p-average decay of $\widehat{\chi}_{P}$ for some, but not for all, polygons $P$.

This is a consequence of Lemma 3.9, Lemma 3.10, Lemma 3.11 and Lemma 3.12.

Finally, for $2 \leq p \leq \infty$, we have

Proposition 2.5. Let $2 \leq p \leq \infty$, then the following are equivalent:

j) There exists a convex body $B$ with piecewise $C^{\infty}$ boundary such that the p-average decay of $\widehat{\chi}_{B}$ has optimal order $a$.

jj) $1+1 / p \leq a \leq 3 / 2$.

The above Proposition follows from Lemma 3.2, Lemma 3.13 and Lemma 3.16.

\section{Lemmas.}

The following lemma is contained in [13, p. 63].

Lemma 3.1. Let $B$ be a convex body in $\mathbb{R}^{2}$. Then

$$
\left(\int_{0}^{2 \pi}\left|\widehat{\chi}_{B}(\rho \Theta)\right|^{2} d \theta\right)^{1 / 2} \leq c \rho^{-3 / 2}
$$

We now prove the following result. 
Lemma 3.2. Let $B$ be a convex body in $\mathbb{R}^{2}$ with piecewise $C^{\infty}$ boundary $\partial B$. Assume $B$ is not a polygon then, for any $p \geq 1$,

$$
\limsup _{\rho \rightarrow \infty} \rho^{3 / 2}\left(\int_{0}^{2 \pi}\left|\widehat{\chi}_{B}(\rho \Theta)\right|^{p} d \theta\right)^{1 / p}>0 \text {. }
$$

Proof. It is enough to prove the lemma when $p=1$. Let $\Gamma$ be an arc in $\partial B$ where the curvature is strictly positive. We examine two cases.

i) There exists an open interval $U$ of angles $\theta$ such that for every $\theta \in U$ there is exactly one point $\sigma(\theta) \in \Gamma$ whose tangent is orthogonal to $\Theta=(\cos \theta, \sin \theta)$ (this may happen since $\partial B$ is only piecewise smooth).

ii) There exists an open interval $U$ of angles $\theta$ such that for every $\theta \in U$ there are exactly two points $\sigma_{1}(\theta), \sigma_{2}(\theta) \in \partial B$ whose tangent is orthogonal to $\Theta$.

We proceed with the proof in case i).

We apply [1, Theorem 1] (see also [13]) to obtain

$$
\widehat{\chi}_{B}(\rho \Theta)=-\frac{1}{2 \pi i} \rho^{-3 / 2} e^{-2 \pi i \rho \Theta \cdot \sigma(\theta)+\pi i / 4} K^{-1 / 2}(\sigma(\theta))+E_{\rho},
$$

where $K(P)$ denotes the curvature at $P \in \partial B$ and $\left|E_{\rho}\right| \leq c \rho^{-2}$. We remark that although [1, Theorem 1] is stated for sets with smooth boundary, in the bidimensional case it still holds true for sets having a piecewise smooth boundary. From (3.1) we have

$$
\rho^{3 / 2} \int_{0}^{2 \pi}\left|\widehat{\chi}_{B}(\rho \Theta)\right| d \theta \geq \frac{1}{2 \pi} \int_{U} K^{-1 / 2}(\sigma(\theta)) d \theta-c_{1} \rho^{-1 / 2} \geq c_{2}>0
$$

We now turn to ii).

As in the previous case we obtain

$$
\widehat{\chi}_{B}(\rho \Theta)=-\frac{1}{2 \pi i} \rho^{-3 / 2} \sum_{j=1}^{2} e^{-2 \pi i \rho \Theta \cdot \sigma_{j}(\theta)+\pi i / 4} K^{-1 / 2}\left(\sigma_{j}(\theta)\right)+E_{\rho} .
$$

We consider three subcases.

a) Suppose first there exists a neighborhood $\widetilde{U} \subseteq U$ where

$$
K\left(\sigma_{1}(\theta)\right) \neq K\left(\sigma_{2}(\theta)\right)
$$


Then

$$
\begin{aligned}
& \rho^{3 / 2} \int_{0}^{2 \pi}\left|\widehat{\chi}_{B}(\rho \Theta)\right| d \theta \\
& \quad \geq \frac{1}{2 \pi} \int_{\widetilde{U}}\left|K^{-1 / 2}\left(\sigma_{1}(\theta)\right)-K^{-1 / 2}\left(\sigma_{2}(\theta)\right)\right| d \theta-c_{1} \rho^{-1 / 2} \geq c_{2}>0
\end{aligned}
$$

b) Suppose there exists a neighborhood $\widetilde{U} \subseteq U$ where the vectors $\Theta$ and $\sigma_{2}(\theta)-\sigma_{1}(\theta)$ are not parallel. Let $A_{j}(\theta)=K^{-1 / 2}\left(\sigma_{j}(\theta)\right)$. We have

$$
\begin{aligned}
\rho^{3 / 2} \int_{0}^{2 \pi} & \left|\widehat{\chi}_{B}(\rho \Theta)\right| d \theta \\
& \geq \frac{1}{2 \pi} \int_{\widetilde{U}}\left|\sum_{j=1}^{2} e^{-2 \pi i \rho \Theta \cdot \sigma_{j}(\theta)} A_{j}(\theta)\right| d \theta-c_{1} \rho^{-1 / 2} \\
& =\frac{1}{2 \pi} \int_{\widetilde{U}}\left|A_{1}(\theta)+A_{2}(\theta) e^{-2 \pi i \rho \Theta \cdot\left(\sigma_{2}(\theta)-\sigma_{1}(\theta)\right)}\right| d \theta-c_{1} \rho^{-1 / 2} \\
& \geq \frac{1}{2 \pi}\left|\int_{\widetilde{U}}\left(A_{1}(\theta)+A_{2}(\theta) e^{-2 \pi i \rho \Theta \cdot\left(\sigma_{2}(\theta)-\sigma_{1}(\theta)\right)}\right) d \theta\right|-c_{1} \rho^{-1 / 2} \\
& \geq M-\frac{1}{2 \pi}\left|\int_{\widetilde{U}} A_{2}(\theta) e^{-2 \pi i \rho \Theta \cdot\left(\sigma_{2}(\theta)-\sigma_{1}(\theta)\right)} d \theta\right|-c_{1} \rho^{-1 / 2}
\end{aligned}
$$

We claim that the last integral tends to zero as $\rho$ tends to infinity. Observe that $\Theta \cdot \sigma_{j}^{\prime}(\theta)=0$ since $\Theta$ is normal to $\partial B$ at the point $\sigma_{j}(\theta)$. Hence

$$
\frac{d}{d \theta}\left(\Theta \cdot\left(\sigma_{2}(\theta)-\sigma_{1}(\theta)\right)\right)=(-\sin \theta, \cos \theta) \cdot\left(\sigma_{2}(\theta)-\sigma_{1}(\theta)\right)
$$

is different from zero since $(-\sin \theta, \cos \theta)$ is not orthogonal to $\sigma_{2}(\theta)-$ $\sigma_{1}(\theta)$. Integration by parts shows that the integral vanishes as $\rho \longrightarrow$ $+\infty$.

c) We suppose now that for every $\theta \in U$ the points $\sigma_{1}(\theta), \sigma_{2}(\theta)$ have the same curvature and that $\Theta$ and $\sigma_{2}(\theta)-\sigma_{1}(\theta)$ are parallel. In this case the quantity (3.2) vanishes so that

$$
\lambda=\Theta \cdot\left(\sigma_{2}(\theta)-\sigma_{1}(\theta)\right)
$$


is constant. Let $K(\theta)=K\left(\sigma_{1}(\theta)\right)=K\left(\sigma_{2}(\theta)\right)$, then

$$
\begin{aligned}
\rho^{3 / 2} \int_{0}^{2 \pi}\left|\widehat{\chi}_{B}(\rho \Theta)\right| d \theta & \geq \frac{1}{2 \pi} \int_{\widetilde{U}} K^{-1 / 2}(\theta)\left|1+e^{-2 \pi i \rho \lambda}\right| d \theta-c_{1} \rho^{-1 / 2} \\
& \geq \frac{1}{2 \pi}\left|1+e^{-2 \pi i \rho \lambda}\right| \int_{\widetilde{U}} K^{-1 / 2}(\theta) d \theta-c_{1} \rho^{-1 / 2}
\end{aligned}
$$

and since

$$
\limsup _{\rho \rightarrow+\infty}\left|1+e^{-2 \pi i \rho \lambda}\right|>0
$$

the proof is complete.

The result of the previous lemma can be strengthened under simple geometric hypothesis on the boundary. The following definition may be useful.

Definition 3.3. We say that a convex body $B$ is a cut disc if it is not a polygon and if its boundary $\partial B$ is the union of a finite number of segments and of a finite number of couples of antipodal arcs of a given circle.

We now need a technical lemma.

Lemma 3.4. Let $I$ and $J$ be two neighborhoods of the origin in $\mathbb{R}$ and let $f \in C^{2}(I), g \in C^{2}(J)$. Assume $f(x)<0, f^{\prime \prime}(x)>0$, for $x \in I, g(x)>0, g^{\prime \prime}(x)<0$ for $x \in J$; also suppose $f(0)=-1$, $g(0)=1, f^{\prime}(0)=g^{\prime}(0)=0$. Finally we assume the existence of $a$ bijection $H: I \longrightarrow J$ such that

i) $f^{\prime}(x)=g^{\prime}(H(x))$,

ii) the curvature of the graph of $f$ at $(x, f(x))$ equals the curvature of the graph of $g$ at $(H(x), g(H(x)))$,

iii) the segment joining the points $(x, f(x))$ and $(H(x), g(H(x)))$ is orthogonal to the tangent lines at these points.

Then the graphs of $f$ and $g$ are two (antipodal) arcs of equal length in the same circle.

ProOF. By our assumptions,

i) $f^{\prime}(x)=g^{\prime}(H(x))$, 
ii) $\frac{f^{\prime \prime}(x)}{\left(1+\left(f^{\prime}(x)\right)^{2}\right)^{3 / 2}}=\frac{-g^{\prime \prime}(H(x))}{\left(1+\left(g^{\prime}(H(x))\right)^{2}\right)^{3 / 2}}$,

iii) $(x-H(x))+(f(x)-g(H(x))) f^{\prime}(x)=0$.

Then i) and ii) imply $f^{\prime \prime}(x)=-g^{\prime \prime}(H(x))$, while differentiating i) one gets $f^{\prime \prime}(x)=g^{\prime \prime}(H(x)) H^{\prime}(x)$. Because of the other assumptions, this implies $H(x)=-x$ and

$$
f(x)=-g(-x)
$$

Then iii) becomes

$$
2 x+2 f(x) f^{\prime}(x)=0,
$$

which gives the equation of a circle.

Lemma 3.4 can be restated in the following, more geometrical, way.

Lemma 3.5. Suppose $B$ is a convex body with piecewise $C^{\infty}$ boundary which is not a cut disc, then $\partial B$ contains a regular point $P$ with unit exterior normal $\Theta$ such that either there is no other regular point in $\partial B$ with unit exterior normal $-\Theta$, or, if such a point $Q$ exists, at least one of the following facts happens: i) $P-Q$ is not parallel to $\Theta$, ii) the curvatures of $\partial B$ at $P$ and at $Q$ differ.

The following is a strengthened version of Lemma 3.2.

Lemma 3.6. Suppose $B$ is a convex body with piecewise $C^{\infty}$ boundary which is neither a polygon nor a cut disc, then, for $1 \leq p \leq 2$,

$$
c_{1} \rho^{-3 / 2} \leq\left(\int_{0}^{2 \pi}\left|\widehat{\chi}_{B}(\rho \Theta)\right|^{p} d \theta\right)^{1 / p} \leq c_{2} \rho^{-3 / 2}
$$

Proof. The estimate from above is contained in Lemma 3.1. On the other hand the estimate from below holds in cases i), ii)-a, ii)-b of the proof of Lemma 3.2. Our assumptions and Lemma 3.5 exclude the case ii)-c. This ends the proof .

The forthcoming lemma is probably known. However, since we have not found a suitable reference, we provide an elementary argument. 
Lemma 3.7. Let $f: \mathbb{R} \longrightarrow[0,+\infty)$ be supported and concave in $[-1,1]$. Then, for every $|\xi| \geq 1$,

$$
|\widehat{f}(\xi)| \leq \frac{1}{|\xi|}\left(f\left(1-\frac{1}{2|\xi|}\right)+f\left(-1+\frac{1}{2|\xi|}\right)\right) .
$$

Proof. It is enough to prove (3.3) when $\xi>1$. The assumption on the concavity of $f$ allows us to integrate by parts obtaining

$$
|\widehat{f}(\xi)| \leq \frac{1}{2 \pi \xi} f\left(1^{-}\right)+\frac{1}{2 \pi \xi} f\left(-1^{+}\right)+\frac{1}{2 \pi \xi}\left|\int_{-1}^{1} f^{\prime}(t) e^{-2 \pi i \xi t} d t\right|
$$

Let $\alpha$ be a point where $f$ attains its maximum. Then $f$ will be nondecreasing in $[-1, \alpha]$ and non-increasing in $[\alpha, 1]$. We can assume $0 \leq$ $\alpha \leq 1$, so that $f\left(-1^{+}\right) \leq f(-1+1 /(2 \xi))$. To estimate $f\left(1^{-}\right)$we observe that when $\alpha \leq 1-1 /(2 \xi)$, one has $f\left(1^{-}\right) \leq f(1-1 /(2 \xi))$. On the other hand, since $f$ is concave, in case $\alpha>1-1 /(2 \xi)$ we have

$$
f\left(1^{-}\right) \leq f(\alpha) \leq 2 f(0) \leq 2 f\left(1-\frac{1}{2 \xi}\right)
$$

To estimate the integral we observe that, by a change of variable,

$$
I=\int_{-1}^{1} f^{\prime}(t) e^{-2 \pi i \xi t} d t=-\int_{-1+1 /(2 \xi)}^{1+1 /(2 \xi)} f^{\prime}\left(t-\frac{1}{2 \xi}\right) e^{-2 \pi i \xi t} d t .
$$

So that

$$
\begin{aligned}
2 I= & \int_{-1}^{1} f^{\prime}(t) e^{-2 \pi i \xi t} d t-\int_{-1+1 /(2 \xi)}^{1+1 /(2 \xi)} f^{\prime}\left(t-\frac{1}{2 \xi}\right) e^{-2 \pi i \xi t} d t \\
= & \int_{-1}^{-1+1 /(2 \xi)} f^{\prime}(t) e^{-2 \pi i \xi t} d t \\
& +\int_{-1+1 /(2 \xi)}^{1}\left(f^{\prime}(t)-f^{\prime}\left(t-\frac{1}{2 \xi}\right)\right) e^{-2 \pi i \xi t} d t \\
& +\int_{1}^{1+1 /(2 \xi)} f^{\prime}\left(t-\frac{1}{2 \xi}\right) e^{-2 \pi i \xi t} d t \\
= & I_{1}+I_{2}+I_{3} .
\end{aligned}
$$


To estimate $I_{1}$ from above we note that

$\left|I_{1}\right| \leq \int_{-1}^{-1+1 /(2 \xi)} f^{\prime}(t) d t=f\left(-1+\frac{1}{2 \xi}\right)-f\left(-1^{+}\right) \leq f\left(-1+\frac{1}{2 \xi}\right)$

since $0 \leq \alpha \leq 1$.

The estimate for $I_{3}$ is similar in case $\alpha \leq 1-1 /(2 \xi)$. If $\alpha>$ $1-1 /(2 \xi)$, then

$$
\begin{aligned}
\left|I_{3}\right| & \leq \int_{1}^{\alpha+1 /(2 \xi)} f^{\prime}\left(t-\frac{1}{2 \xi}\right) d t-\int_{\alpha+1 /(2 \xi)}^{1+1 /(2 \xi)} f^{\prime}\left(t-\frac{1}{2 \xi}\right) d t \\
& =2 f(\alpha)-f\left(1-\frac{1}{2 \xi}\right)-f\left(1^{-}\right) \\
& \leq 2 f(\alpha) \\
& \leq 4 f(0) \\
& \leq 4 f\left(1-\frac{1}{2 \xi}\right) .
\end{aligned}
$$

As for $I_{2}$, since $f^{\prime}$ is non increasing, we have

$$
\begin{aligned}
\left|I_{2}\right| & \leq \int_{-1+1 /(2 \xi)}^{1}\left(f^{\prime}\left(t-\frac{1}{2 \xi}\right)-f^{\prime}(t)\right) d t \\
& =f\left(1-\frac{1}{2 \xi}\right)-f\left(-1^{+}\right)-f\left(1^{-}\right)+f\left(-1+\frac{1}{2 \xi}\right) \\
& \leq f\left(1-\frac{1}{2 \xi}\right)+f\left(-1+\frac{1}{2 \xi}\right)
\end{aligned}
$$

ending the proof. Note that no constant $c$ is missing in (3.3).

REMARK. A different proof of the above lemma can be modeled on an argument similar to that of Lemma 3.15 below.

The following result is similar to [5, Theorem 6.1] (see also [13, Lemma 3]). Our proof is based on the previous lemma.

Lemma 3.8. Let $B$ be a convex body in $\mathbb{R}^{2}, \Theta=(\cos \theta, \sin \theta)$ and $S_{\theta}=\sup _{x \in B} x \cdot \Theta$. For $\rho \geq 1$ we set (see Figure 2 with $\rho^{-1}$ in place of $\delta)$

$$
A_{B}\left(\rho^{-1}, \theta\right)=\left\{x \in B: S_{\theta}-\rho^{-1} \leq x \cdot \Theta \leq S_{\theta}\right\} .
$$


Then

$$
\left|\widehat{\chi}_{B}(\rho \Theta)\right| \leq c\left(\left|A_{B}\left(\rho^{-1}, \theta\right)\right|+\left|A_{B}\left(\rho^{-1}, \theta+\pi\right)\right|\right),
$$

where $|E|$ denotes the Lebesgue measure of a measurable set $E$.

Proof. Without loss of generality we choose $\Theta=(1,0)$. Then

$$
\begin{aligned}
\widehat{\chi}_{B}\left(\xi_{1}, 0\right) & =\int_{-\infty}^{+\infty}\left(\int_{-\infty}^{+\infty} \chi_{B}\left(x_{1}, x_{2}\right) d x_{2}\right) e^{-2 \pi i x_{1} \xi_{1}} d x_{1} \\
& =\widehat{h}\left(\xi_{1}\right)
\end{aligned}
$$

where $h(s)$ is the lenght of the segment obtained intersecting $B$ with the line $x_{1}=s$. Observe that $h$ is concave on its support, say $[a, b]$. We can therefore apply Lemma 3.7 to obtain, after a change of variable,

$$
\begin{aligned}
\left|\widehat{h}\left(\xi_{1}\right)\right| & \leq \frac{1}{\left|\xi_{1}\right|}\left(h\left(b-\frac{1}{2\left|\xi_{1}\right|}\right)+h\left(a+\frac{1}{2\left|\xi_{1}\right|}\right)\right) \\
& \leq c\left(\left|A_{B}\left(\left|\xi_{1}\right|^{-1}, 0\right)\right|+\left|A_{B}\left(\left|\xi_{1}\right|^{-1}, \pi\right)\right|\right) .
\end{aligned}
$$

We now consider polygons.

The following lemma appears in [3]; here we give a different, more geometric, argument based on the previous lemma.

Lemma 3.9. Let $P$ be a compact polygon in $\mathbb{R}^{2}$. Then

$$
\left(\int_{0}^{2 \pi}\left|\widehat{\chi}_{P}(\rho \Theta)\right|^{p} d \theta\right)^{1 / p} \leq \begin{cases}c \rho^{-2} \log (1+\rho), & \text { when } p=1 \\ c \rho^{-1-1 / p}, & \text { when } p>1\end{cases}
$$

Proof. Without loss of generality we can assume that the polygon is convex, lies in the left halfplane and that the points $(0,-1)$ and $(0,1)$ are vertices. By Lemma 3.8 we reduce the problem to estimating $\left|A_{P}(1 / \rho, \theta)\right|$ in a suitable right neighborhood of zero. A simple geometric consideration shows that

$$
\left|A_{P}\left(\rho^{-1}, \theta\right)\right| \leq \begin{cases}c \rho^{-1}, & \text { for } 0 \leq \theta \leq c_{1} \rho^{-1} \\ c_{2} \rho^{-1} \theta^{-1}, & \text { for } c_{1} \rho^{-1} \leq \theta \leq c_{3}\end{cases}
$$

which implies (3.5) by integration. 
We still have to check sharpness of the estimates in (3.5). This is not entirely trivial since parallel edges of $P$ (if any) give the same contribution to the decay of $\widehat{\chi}_{P}$ so that cancellations may occur. Actually this does not happen for $p=1$, but it may happen for $p>1$, as shown in the next three lemmas.

Lemma 3.10. Let $\chi_{P}$ be the characteristic function of a compact convex polygon $P$ in $\mathbb{R}^{2}$ with non empty interior. Then

$$
\int_{0}^{2 \pi}\left|\widehat{\chi}_{P}(\rho \Theta)\right| d \theta \geq c \rho^{-2} \log (1+\rho) .
$$

Proof. Let $L_{j}=\left[P_{j}, P_{j+1}\right], j=1, \ldots, S$, be the edges of the polygon $P$ and let $l_{j}$ be their lengths. Then, with the aid of the divergence formula, we obtain

$$
\begin{aligned}
\widehat{\chi}_{P}(\rho \Theta) & =\int_{P} e^{-2 \pi i \rho \Theta \cdot t} d t \\
& =-\frac{1}{2 \pi i \rho} \int_{\partial P} e^{-2 \pi i \rho \Theta \cdot t} \Theta \cdot \nu(t) d t^{\prime} \\
& =\frac{1}{4 \pi^{2} \rho^{2}} \sum_{j=1}^{S} \Theta \cdot \nu_{j} \frac{e^{-2 \pi i \rho \Theta \cdot P_{j+1}}-e^{-2 \pi i \rho \Theta \cdot P_{j}}}{\Theta \cdot\left(P_{j+1}-P_{j}\right)} l_{j},
\end{aligned}
$$

where $d t^{\prime}$ is the 1-dimensional measure and $\nu_{j}$ is the outward unit normal to $L_{j}$. The argument is divided in three cases.

Case 1. Suppose there exists an edge, say $L_{1}$, which is not parallel to any other edge. We can suppose $P_{1}=(0,-1)$ and $P_{2}=(0,1)$.

Because of these assumptions there exists a right neighborhood $U(0) \subset[0,2 \pi)$ such that

$$
\inf _{\theta \in U(0)}\left|\Theta \cdot\left(P_{j+1}-P_{j}\right)\right| \geq c>0,
$$

for each $j \geq 2$. Hence

$$
\begin{aligned}
\int_{0}^{2 \pi}\left|\widehat{\chi}_{P}(\rho \Theta)\right| d \theta & \geq \frac{c_{1}}{\rho^{2}} \int_{U(0)}\left|\Theta \cdot \nu_{1} \frac{e^{-2 \pi i \rho \Theta \cdot P_{2}}-e^{-2 \pi i \rho \Theta \cdot P_{1}}}{\Theta \cdot\left(P_{2}-P_{1}\right)} l_{1}\right| d \theta-\frac{c_{2}}{\rho^{2}} \\
& \geq \frac{c_{3}}{\rho^{2}} \int_{U(0)}\left|\cos \theta \frac{\sin (2 \pi \rho \sin \theta)}{\sin \theta}\right| d \theta-\frac{c_{2}}{\rho^{2}}
\end{aligned}
$$




$$
\begin{aligned}
& \geq \frac{c_{4}}{\rho^{2}} \int_{0}^{c_{5}}\left|\frac{\sin (2 \pi \rho u)}{u}\right| d u-\frac{c_{2}}{\rho^{2}} \\
& \geq c \rho^{-2} \log (1+\rho) .
\end{aligned}
$$

Case 2. Suppose there exists a couple of parallel edges of different length. Let $M_{1}=\left[Q_{1}, R_{1}\right]$ and $M_{2}=\left[Q_{2}, R_{2}\right]$ be such a pair.

We can assume $Q_{1}=H_{1}+\left(0,-a_{1}\right), R_{1}=H_{1}+\left(0, a_{1}\right), Q_{2}=$ $H_{2}+\left(0,-a_{2}\right), R_{2}=H_{2}+\left(0, a_{2}\right)$ with $a_{2}>a_{1}>0$.

Then, arguing as above,

$$
\begin{aligned}
\int_{0}^{2 \pi}\left|\widehat{\chi}_{P}(\rho \Theta)\right| d \theta \\
\quad \geq \frac{c_{1}}{\rho^{2}} \int_{U(0)}\left|\sum_{j=1}^{2} \Theta \cdot \nu_{1} \frac{e^{-2 \pi i \rho \Theta \cdot Q_{j}}-e^{-2 \pi i \rho \Theta \cdot R_{j}}}{\Theta \cdot\left(Q_{j}-R_{j}\right)} l_{j}\right| d \theta-\frac{c_{2}}{\rho^{2}} \\
\quad \geq \frac{c_{1}}{\rho^{2}} \int_{U(0)}\left|\cos \theta \sum_{j=1}^{2} e^{-2 \pi i \rho \Theta \cdot H_{j}} \frac{\sin \left(2 \pi \rho a_{j} \sin \theta\right)}{a_{j} \sin \theta}\right| d \theta-\frac{c_{2}}{\rho^{2}} \\
\quad \geq \frac{c_{3}}{\rho^{2}}\left(\int_{0}^{c_{4}}\left|\frac{\sin \left(2 \pi \rho a_{1} u\right)}{a_{1} u}\right| d u-\int_{0}^{c_{4}}\left|\frac{\sin \left(2 \pi \rho a_{2} u\right)}{a_{2} u}\right| d u\right)-\frac{c_{2}}{\rho^{2}} \\
\geq \frac{c_{3}}{\rho^{2}}\left(\frac{1}{a_{1}} \log \left(a_{1} \rho\right)-\frac{1}{a_{2}} \log \left(a_{2} \rho\right)\right)-\frac{c_{5}}{\rho^{2}} \\
\geq c \rho^{-2} \log (1+\rho) .
\end{aligned}
$$

Case 3. Suppose the edges of $P$ are pairwise parallel and with the same length. Let $M_{1}=\left[Q_{1}, R_{1}\right]$ and $M_{2}=\left[Q_{2}, R_{2}\right]$ be one of these couples. We can assume $Q_{1}=H+(0,-1), R_{1}=H+(0,1), Q_{2}=-H+(0,-1)$, $R_{2}=-H+(0,1)$. Then

$$
\begin{aligned}
\int_{0}^{2 \pi}\left|\widehat{\chi}_{P}(\rho \Theta)\right| d \theta & \\
& \geq \frac{c_{1}}{\rho^{2}} \int_{U(0)}\left|\sum_{j=1}^{2} \Theta \cdot \nu_{1} \frac{e^{-2 \pi i \rho \Theta \cdot Q_{j}}-e^{-2 \pi i \rho \Theta \cdot R_{j}}}{\Theta \cdot\left(Q_{j}-R_{j}\right)}\right| d \theta-\frac{c_{2}}{\rho^{2}} \\
& \geq \frac{c_{1}}{\rho^{2}} \int_{U(0)}\left|\cos (2 \pi \rho \Theta \cdot H) \frac{\sin (2 \pi \rho \sin \theta)}{\sin \theta}\right| d \theta-\frac{c_{2}}{\rho^{2}} .
\end{aligned}
$$


Let $H=\left(h_{1}, h_{2}\right)$ and $\Theta \cdot H=h_{1} \cos \theta+h_{2} \sin \theta$. We choose $\varphi$ so that $\Theta \cdot H=|H| \sin (\theta+\varphi)$. Since $h_{1} \neq 0$ we have $\varphi \neq 0$ and for symmetry reasons we can restrict ourselves to the case $0<\varphi \leq \pi / 2$.

We obtain

$$
\begin{aligned}
& \int_{0}^{2 \pi}\left|\widehat{\chi}_{P}(\rho \Theta)\right| d \theta \\
& \quad \geq \frac{c_{1}}{\rho^{2}} \int_{U(0)}\left|\cos (2 \pi \rho|H| \sin (\theta+\varphi)) \frac{\sin (2 \pi \rho \sin \theta)}{\sin \theta}\right| d \theta-\frac{c_{2}}{\rho^{2}} .
\end{aligned}
$$

Observe that choosing a sequence $\rho_{n}$ so that $\rho_{n}|H| \sin \varphi$ is close to an integer we immediately get

$$
\int_{0}^{2 \pi}\left|\widehat{\chi}_{P}\left(\rho_{n} \Theta\right)\right| d \theta \geq \frac{c}{\rho_{n}^{2}} \int_{U(0)}\left|\frac{\sin \left(2 \pi \rho_{n} \sin \theta\right)}{\sin \theta}\right| d \theta \geq c \rho_{n}^{-2} \log \left(1+\rho_{n}\right),
$$

that is, we have proved that $\rho^{-2} \log (1+\rho)$ is an optimal estimate of the 1-average decay of $\hat{\chi}_{P}$. To get the full statement of the lemma we must deal with the values of $\rho$ close to those annihilating $\cos (2 \pi \rho|H| \sin \varphi)$.

We begin with the case $0<\varphi<\pi / 2$. Let $0<\varepsilon<\pi / 2-\varphi$ such that $[0, \varepsilon] \subseteq U(0)$ and let $\left\{\left[a_{j}, b_{j}\right]\right\}$ be the collection of intervals determined by the choice

$$
a_{j}=\arcsin \left(\frac{j+\frac{1}{2}+\delta}{2 \rho|H|}\right)-\varphi, \quad b_{j}=\arcsin \left(\frac{j+\frac{3}{2}-\delta}{2 \rho|H|}\right)-\varphi
$$

and $j=[2 \rho|H| \sin \varphi]+1, \ldots,[2 \rho|H| \sin (\varphi+\varepsilon)]$ for some sufficiently small $\delta>0$. We observe that on each $\left[a_{j}, b_{j}\right]$ we have

$$
|\cos (2 \pi \rho|H| \sin (\theta+\varphi))| \geq \delta^{\prime}>0 \text {. }
$$

As a consequence

$$
\begin{aligned}
\int_{U(0)} \mid \cos (2 \pi \rho|H| & \sin (\theta+\varphi)) \frac{\sin (2 \pi \rho \sin \theta)}{\sin \theta} \mid d \theta \\
& \geq \delta^{\prime} \sum_{j} \frac{1}{\sin b_{j}} \int_{a_{j}}^{b_{j}}|\sin (2 \pi \rho \sin \theta)| d \theta \\
& \geq c \delta^{\prime} \sum_{j} \frac{1}{\rho \sin b_{j}} \int_{\rho \sin a_{j}}^{\rho \sin b_{j}}|\sin (2 \pi u)| d u .
\end{aligned}
$$


Using the elementary inequality

$$
\sin \left(b_{j}+\varphi\right)-\sin \left(a_{j}+\varphi\right) \leq \sin b_{j}-\sin a_{j}
$$

and the above definition of $a_{j}$ and $b_{j}$ we see that the quantity

$$
\rho \sin b_{j}-\rho \sin a_{j}
$$

is bounded away from zero and therefore

$$
\int_{\rho \sin a_{j}}^{\rho \sin b_{j}}|\sin (2 \pi u)| d u>c>0 .
$$

Now the choice of $b_{j}$ implies

$$
\sum_{j} \frac{1}{\rho \sin b_{j}} \geq c \log (1+\rho) .
$$

Indeed, let $k=j-[2 \rho|H| \sin \varphi]$, so that we have to estimate

$$
\sum_{k=1}^{c \rho} \frac{1}{\rho \sin b_{k+[2 \rho|H| \sin \varphi]}}
$$

from below. The choice of $b_{j}$ shows that

$$
\sin b_{k+[2 \rho|H| \sin \varphi]} \leq \frac{k+2}{2 \rho|H|}
$$

and therefore the last term in (3.6) is greater than

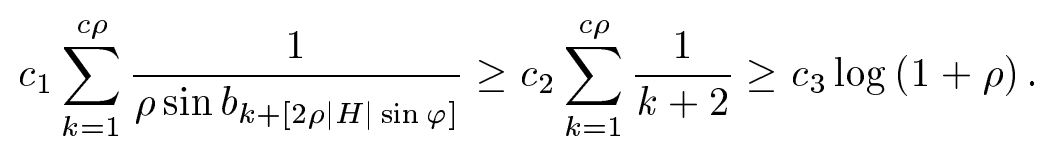

The case $\varphi=\pi / 2$ is similar. We fix $\varepsilon>0$ so that $[0, \varepsilon] \subset U(0)$. Next, we consider the collection of intervals $\left\{\left[a_{j}, b_{j}\right]\right\}$ with

$$
a_{j}=\frac{\pi}{2}-\arcsin \left(\frac{j+\frac{1}{2}+\delta}{2 \rho|H|}\right), \quad b_{j}=\frac{\pi}{2}-\arcsin \left(\frac{j+\frac{3}{2}-\delta}{2 \rho|H|}\right)
$$


and $j=[2 \rho|H| \sin (\pi / 2+\varepsilon)+1], \ldots,[2 \rho|H|]$ for some sufficiently small $\delta>0$. As before on each $\left[a_{j}, b_{j}\right]$ we have

$$
|\cos (2 \pi \rho|H| \sin (\theta+\varphi))| \geq \delta^{\prime}>0 \text {. }
$$

Using the fact that

$$
\frac{\pi}{2}-\arcsin x=2 \arcsin \sqrt{\frac{1-x}{2}}
$$

one deduces the estimates

$$
a_{j} \approx \sqrt{\frac{2 \rho|H|-j-\frac{1}{2}-\delta}{\rho|H|}}, \quad b_{j} \approx \sqrt{\frac{2 \rho|H|-j-\frac{3}{2}+\delta}{\rho|H|}}
$$

and consequently the required result.

Lemma 3.11. Let $\chi_{P}$ be the characteristic function of a compact polygon $P$ in $\mathbb{R}^{2}$. For any $p>1$

$$
\limsup _{\rho \rightarrow \infty} \rho^{1+1 / p}\left(\int_{0}^{2 \pi}\left|\widehat{\chi}_{P}(\rho \Theta)\right|^{p} d \theta\right)^{1 / p}>0 .
$$

Proof. We can suppose that one of the sides of $P$ is vertical. We assume the following facts, which will be proved in the sequel:

$$
\begin{aligned}
& \text { there exists } \rho_{k} \longrightarrow+\infty \text { so that }\left|\widehat{\chi}_{P}\left(\rho_{k}, 0\right)\right| \geq \frac{c}{\rho_{k}} \\
& \qquad\left|\nabla \widehat{\chi}_{P}(\xi)\right| \leq \frac{c}{|\xi|+1} .
\end{aligned}
$$

Next we consider

$$
\int_{0}^{2 \pi}\left|\widehat{\chi}_{P}\left(\rho_{k} \Theta\right)\right|^{p} d \theta \geq \int_{0}^{\varepsilon / \rho_{k}}\left|\widehat{\chi}_{P}\left(\rho_{k} \Theta\right)\right|^{p} d \theta
$$

By choosing $\varepsilon$ sufficiently small we can make $\left(\rho_{k} \cos \theta, \rho_{k} \sin \theta\right)$ close to $\left(\rho_{k}, 0\right)$ so that $(3.8),(3.7)$ and the mean value theorem imply

$$
\left|\widehat{\chi}_{P}\left(\rho_{k} \Theta\right)\right| \geq \frac{c_{1}}{\rho_{k}} .
$$


Hence,

$$
\int_{0}^{2 \pi}\left|\widehat{\chi}_{P}\left(\rho_{k} \Theta\right)\right|^{p} d \theta \geq c \int_{0}^{\varepsilon / \rho_{k}} \rho_{k}^{-p} d \theta \geq c \rho_{k}^{-p-1} .
$$

We now prove (3.7).

First we recall, see (3.4), that

$$
\widehat{\chi}_{P}\left(\xi_{1}, 0\right)=\widehat{h}\left(\xi_{1}\right)
$$

where $h(t)$ is the length of the chord given by the intersection of $P$ with the line $x_{1}=t$. Observe that $h(t)$ is a piecewise linear function, continuous at any point except at least one of the extremes of the support. Split

$$
h(t)=b(t)+g(t)
$$

where $b(t), g(t)$ and $h(t)$ share the same support, $b(t)$ is linear inside the support and $g(t)$ is continuous on $\mathbb{R}$. Our choice forces $b(t)$ to be discontinuous in at least one of the extremes (recall that at least one side of $P$ is ortogonal to $(1,0)$ ), while $g(t)$ must be piecewise linear.

An explicit computation gives a sequence $\rho_{k} \longrightarrow+\infty$ such that $\left|\widehat{b}\left(\rho_{k}\right)\right| \geq c \rho_{k}^{-1}$, while

$$
\left|\widehat{g}\left(\xi_{1}\right)\right| \leq c \frac{1}{1+\xi_{1}^{2}}
$$

This proves (3.7).

In order to prove (3.8) we observe that, for any unit vector $u$,

$$
\begin{aligned}
\frac{\partial}{\partial u} \widehat{\chi}_{P}(\xi) & =\frac{\partial}{\partial u} \int_{P} e^{-2 \pi i \xi \cdot x} d x \\
& =-2 \pi i \int_{P}(u \cdot x) e^{-2 \pi i \xi \cdot x} d x \\
& =-2 \pi i \int_{\mathbb{R}^{2}}\left((u \cdot x) \chi_{P}(x)\right) e^{-2 \pi i \xi \cdot x} d x
\end{aligned}
$$

and (3.8) follows since the function $x \longrightarrow(u \cdot x) \chi_{P}(x)$ has bounded variation.

The following lemma is taken from [3]. We reproduce the short proof. 
Lemma 3.12. i) Let $P$ be a polygon having an edge not parallel to any other. Then, if $1 \leq p \leq \infty$,

$$
\left(\int_{0}^{2 \pi}\left|\widehat{\chi}_{Q}(\rho \Theta)\right|^{p} d \theta\right)^{1 / p} \geq c \rho^{-1-1 / p} .
$$

ii) Let $Q$ be the unit square $[-1 / 2,1 / 2]^{2}$. If $1<p \leq+\infty$ and if $k$ is a positive integer, then

$$
\left(\int_{0}^{2 \pi}\left|\widehat{\chi}_{Q}(k \Theta)\right|^{p} d \theta\right)^{1 / p} \leq c k^{-3 / 2-1 /(2 p)}
$$

Proof. i) Arguing as in the first case in the proof of Lemma 3.10 we are reduced to bounding

$$
\frac{1}{\rho^{2 p}} \int_{0}^{c_{1}}\left|\frac{\sin (2 \pi \rho u)}{u}\right|^{p} d u
$$

from below. A computation ends the proof of this case.

ii) We have

$$
\begin{aligned}
\int_{0}^{2 \pi}\left|\widehat{\chi}_{Q}(k \Theta)\right|^{p} d \theta & =8 \int_{0}^{\pi / 4}\left|\frac{\sin (\pi k \cos \theta)}{\pi k \cos \theta} \frac{\sin (\pi k \sin \theta)}{\pi k \sin \theta}\right|^{p} d \theta \\
& \leq c k^{-2 p} \int_{0}^{\pi / 4}\left|\frac{\sin (\pi k \cos \theta)}{\sin \theta}\right|^{p} d \theta \\
& \leq c k^{-2 p} \int_{0}^{\pi / 4}\left|\sin \left(2 \pi k \sin ^{2}\left(\frac{\theta}{2}\right)\right)\right|^{p} \theta^{-p} d \theta \\
& \leq c k^{-2 p} \int_{0}^{k^{-1 / 2}} k^{p} \theta^{p} d \theta+c k^{-2 p} \int_{k^{-1 / 2}}^{\pi / 4} \theta^{-p} d \theta \\
& \leq c k^{-3 p / 2-1 / 2} .
\end{aligned}
$$

The forthcoming results will be used in the proof of Proposition 2.5 .

Lemma 3.13. Let $2 \leq p \leq+\infty$ and let $s<1+1 / p$. Then the $p$ average decay of $\hat{\chi}_{B}$ has optimal order $s$ for no convex body $B$ with piecewise $C^{\infty}$ boundary. 
Proof. Lemma 3.1 and the theorem on the decay of the Fourier transform of a function of bounded variation imply this lemma when $p=2$ and $p=\infty$ respectively. When $2<p<\infty$ we have

$$
\begin{aligned}
\left(\int_{0}^{2 \pi}\left|\widehat{\chi}_{B}(\rho \Theta)\right|^{p} d \theta\right)^{1 / p} & =\left(\int_{0}^{2 \pi}\left|\widehat{\chi}_{B}(\rho \Theta)\right|^{2}\left|\widehat{\chi}_{B}(\rho \Theta)\right|^{p-2} d \theta\right)^{1 / p} \\
& \leq c\left(\int_{0}^{2 \pi}\left|\widehat{\chi}_{B}(\rho \Theta)\right|^{2} d \theta\right)^{1 / p} \rho^{-1+2 / p} \\
& \leq c \rho^{-1-1 / p} .
\end{aligned}
$$

Lemma 3.14. Let $P=\left(s_{0}, s_{0}^{\alpha}\right)$ be a given point in the graph of the function $t=s^{\alpha}$, with $0<\alpha<1$. Let $\varphi=\arctan \left(\alpha s_{0}^{\alpha-1}\right)$ be the slope of the corresponding tangent line and let, for a small positive $\delta$,

$$
t=\alpha s_{0}^{\alpha-1}\left(s-s_{0}\right)+s_{0}^{\alpha}-\frac{\delta}{\cos \varphi}
$$

be parallel to the above tangent line, at distance $\delta$. Here we assume that this last line and the curve $t=s^{\alpha}$ intersect in two points $A=\left(s_{1}, s_{1}^{\alpha}\right)$ and $B=\left(s_{2}, s_{2}^{\alpha}\right)$ (see Figure 3$)$. We denote by $d(\delta)$ the distance between $A$ and $B$. Then $d^{\prime}(\delta)$ is a convex function of $\delta$.

Figure 3. 
Proof. Since $d(\delta)=\left(s_{2}-s_{1}\right) / \cos \varphi$ it is enough to check that the functions $h(\delta)=s_{2}-s_{0}$ and

$$
k(\delta)=\frac{s_{0}-s_{1}}{\cos \varphi}+\delta \tan \varphi
$$

have convex derivatives.

We start with $h(\delta)$. By the definition of the point $B$ we have

$$
s_{2}^{\alpha}=\alpha s_{0}^{\alpha-1}\left(s_{2}-s_{0}\right)+s_{0}^{\alpha}-\frac{\delta}{\cos \varphi},
$$

that is

$$
\left(h(\delta)+s_{0}\right)^{\alpha}=\alpha s_{0}^{\alpha-1} h(\delta)+s_{0}^{\alpha}-\frac{\delta}{\cos \varphi} .
$$

Differentiating the above with respect to $\delta$ we get

$$
\alpha\left(h(\delta)+s_{0}\right)^{\alpha-1} h^{\prime}(\delta)=\alpha s_{0}^{\alpha-1} h^{\prime}(\delta)-\frac{1}{\cos \varphi},
$$

which implies $h^{\prime}(\delta)>0$ since $0<\alpha<1$. Further differentiations show that $h^{\prime \prime}(\delta)<0$ and $h^{\prime \prime \prime}(\delta)>0$.

We now turn to $k(\delta)$, which is the distance between the points $A$ and $C$ in Figure 3. In order to prove that the negative function $k^{\prime \prime}(\delta)$ increases with $\delta$ we observe that

$$
k^{\prime \prime}(\delta)=-K(A)\left(1+\left(k^{\prime}(\delta)\right)^{2}\right)^{3 / 2},
$$

where $K(A)$ denotes the curvature at the point $A$. Now it is easy to check that $K(A)$ decreases as $A$ moves towards $O$ (that is as $\delta$ grows). On the other hand, by convexity, $k^{\prime}(\delta)$ decreases too. Therefore, by $(3.10), k^{\prime \prime}(\delta)$ increases and this ends the proof of the lemma.

The following result is related to [5, Lemmas 6.2 and 6.3$]$.

Lemma 3.15. Let $f: \mathbb{R} \longrightarrow \mathbb{R}^{+}$be supported in $[-1,1]$, such that $f \in C^{\infty}(\mathbb{R} \backslash\{1\}), f \in C(\mathbb{R}), f$ and $f^{\prime}$ are concave in $[b, 1)$ and $f^{\prime}(b)=0$, $f^{\prime}\left(1^{-}\right)=-\infty$. Then, for $|\xi| \geq 1$,

$$
|\widehat{f}(\xi)| \geq c \frac{1}{|\xi|} f\left(1-\frac{1}{6|\xi|}\right) .
$$


The constant $c$ depends only on the supremum of $|f(t)|$ on $\mathbb{R}$ and on the variation of $f^{\prime}(t)$ outside a neighborhood of $t=1$.

Proof. We write

$$
\begin{aligned}
\widehat{f}(\xi) & =\int_{-1}^{1} f(t) e^{-2 \pi i t \xi} d t \\
& =\frac{1}{2 \pi i \xi} \int_{-1}^{1} f^{\prime}(t) e^{-2 \pi i t \xi} d t \\
& =\frac{1}{2 \pi i \xi} \int_{-1}^{b} f^{\prime}(t) e^{-2 \pi i t \xi} d t+\frac{1}{2 \pi i \xi} \int_{b}^{1} f^{\prime}(t) e^{-2 \pi i t \xi} d t \\
& =I_{1}(\xi)+I_{2}(\xi) .
\end{aligned}
$$

Since $f^{\prime}$ is of bounded variation on $[-1, b]$ we have $\left|I_{1}(\xi)\right| \leq c|\xi|^{-2}$ where $c$ depends only on the variation of $f^{\prime}$. Morover, $f$ concave on $[b, 1]$ and $f^{\prime}\left(1^{-}\right)=-\infty$ imply

$$
|\xi|^{-2}=o\left(|\xi|^{-1} f\left(1-\frac{1}{6|\xi|}\right)\right)
$$

so that

$$
\left|I_{1}(\xi)\right|=o\left(|\xi|^{-1} f\left(1-\frac{1}{6|\xi|}\right)\right)
$$

To analyze $I_{2}(\xi)$ we proceed as follows: we assume $\xi>0$ (the case $\xi<0$ is similar) we write $\xi=[\xi]+\eta$ and let $\sigma=(1-6 \eta) /(6 \xi)$ (this choice will be appreciated later on, while estimating $\left.I_{5}(\xi)\right)$. Then

$$
\begin{aligned}
\left|I_{2}(\xi)\right| & =\frac{1}{2 \pi \xi}\left|\int_{b}^{1}\left(-f^{\prime}(t)\right) e^{2 \pi i t \xi} d t\right| \\
& =\frac{1}{2 \pi \xi}\left|\int_{b}^{1}\left(-f^{\prime}(t)\right) e^{2 \pi i(t+\sigma) \xi} d t\right| \\
& \geq \frac{1}{2 \pi \xi}\left|\int_{b}^{1}\left(-f^{\prime}(t)\right) \cos (2 \pi(t+\sigma) \xi) d t\right| \\
& =\frac{1}{2 \pi \xi}\left|I_{3}(\xi)+I_{4}(\xi)+I_{5}(\xi)\right|,
\end{aligned}
$$


where

$$
\begin{gathered}
I_{3}(\xi)=\int_{b}^{j_{0} /(4 \xi)-\sigma}\left(-f^{\prime}(t)\right) \cos (2 \pi(t+\sigma) \xi) d t, \\
I_{4}(\xi)=\sum_{j=j_{0}}^{4[\xi]-1} \int_{j /(4 \xi)-\sigma}^{(j+1) /(4 \xi)-\sigma}\left(-f^{\prime}(t)\right) \cos (2 \pi(t+\sigma) \xi) d t=\sum_{j=j_{0}}^{4[\xi]-1} A_{j}, \\
I_{5}(\xi)=\int_{1-1 /(6 \xi)}^{1}\left(-f^{\prime}(t)\right) \cos (2 \pi(t+\sigma) \xi) d t,
\end{gathered}
$$

with $j_{0}$ the smallest even integer such that $j_{0} /(4 \xi)-\sigma \geq b$. First we observe that $\left|I_{3}(\xi)\right| \leq c / \xi$ and therefore its contribution is negligible.

We consider $I_{4}(\xi)$ and we show that

$$
I_{4}(\xi)=\sum_{j=j_{0}}^{4[\xi]-1} A_{j} \geq 0
$$

Indeed,

i) $A_{4[\xi]-1}>0, A_{4[\xi]-2}<0, A_{4[\xi]-3}<0, A_{4[\xi]-4}>0, A_{4[\xi]-5}>0$, $A_{4[\xi]-6}<0, \ldots$

ii) $\left|A_{j}\right| \leq\left|A_{j+1}\right|$ so that $A_{4[\xi]-1}+A_{4[\xi]-2}>0, A_{4[\xi]-3}+A_{4[\xi]-4}<0$, $A_{4[\xi]-5}+A_{4[\xi]-6}>0, \ldots$ $\geq \cdots$

iii) $\left|A_{4[\xi]-1}+A_{4[\xi]-2}\right| \geq\left|A_{4[\xi]-3}+A_{4[\xi]-4}\right| \geq\left|A_{4[\xi]-5}+A_{4[\xi]-6}\right|$

The validity of $\mathrm{i}$ ) is obvious, while ii) depends on the monotonicity of $f^{\prime}$. As for iii) we note that the concavity of $f^{\prime}$ implies

$$
\left|A_{4[\xi]-1}\right|-\left|A_{4[\xi]-3}\right| \geq\left|A_{4[\xi]-2}\right|-\left|A_{4[\xi]-4}\right| \geq \cdots
$$

By i), ii), iii) it follows that the sum

$$
\left(A_{j_{0}}+A_{j_{0}+1}\right)+\left(A_{j_{0}+2}+A_{j_{0}+3}\right)+\cdots+\left(A_{4[\xi]-2}+A_{4[\xi]-1}\right)
$$

shares the sign of its last term $\left(A_{4[\xi]-2}+A_{4[\xi]-1}\right)$, thereby proving (3.12).

Hence,

$$
I_{4}(\xi)+I_{5}(\xi) \geq I_{5}(\xi)=\int_{1-1 /(6 \xi)}^{1}\left(-f^{\prime}(t)\right) \cos (2 \pi(t+\sigma) \xi) d t \geq \frac{1}{2} f\left(1-\frac{1}{6 \xi}\right)
$$


since $\cos (2 \pi(t+\sigma) \xi) \geq 1 / 2$ on the domain of integration.

Lemma 3.16. For each

$$
\left(\frac{1}{p}, a\right) \in T=\left\{\left(\frac{1}{p}, a\right): 2 \leq p \leq \infty, 1+\frac{1}{p}<a<\frac{3}{2}\right\}
$$

there exists a convex body $B$ with piecewise $C^{\infty}$ boundary such that the p-average decay of $\widehat{\chi}_{B}$ has sharp order a.

Proof. Let $B$ be a convex body symmetric with respect to the vertical axis and assume that its boundary $\partial B$ satisfies the following conditions. point.

i) $\partial B$ passes through the origin and it is of class $C^{\infty}$ in any other

ii) $\partial B$ coincides with the graph of the function $y=|x|^{\gamma}$ in a neighborhood of the origin (the exponent $\gamma=\gamma(p, a)>2$ will be chosen later). hood.

iii) $\partial B$ has strictly positive curvature out of the above neighbor-

We first prove that $\left|\widehat{\chi}_{B}(\rho \Theta)\right| \leq c \rho^{-1-1 / \gamma}$ for any $\Theta \in \Sigma_{1}$. This bound seems to be quite obvious since $\left|\xi_{2}\right|^{-1-1 / \gamma}$ is the order of decay of $\widehat{\chi}_{B}\left(0, \xi_{2}\right)$, that is, the decay associated to the flattest point in $\partial B$. However, a proof seems to be necessary (in order to check that the constant does not depend on $\Theta$ ), and the argument will be needed in the sequel.

Let $\psi=\theta+\pi / 2$. We choose $\varepsilon>0$ sufficiently small and we assume $\varepsilon \leq|\psi| \leq \pi-\varepsilon$. Since $\partial B$ has strictly positive curvature away from the origin, by Lemma 3.8 we have,

$$
\left|\widehat{\chi}_{B}(\rho \Theta)\right| \leq c\left(\left|A_{B}\left(\rho^{-1}, \psi-\frac{\pi}{2}\right)\right|+\left|A_{B}\left(\rho^{-1}, \psi+\frac{\pi}{2}\right)\right|\right) \leq c \rho^{-3 / 2},
$$

for $\varepsilon \leq|\psi| \leq \pi-\varepsilon$.

Symmetry enables us to consider only the case $0 \leq \psi \leq \varepsilon$. The assumptions on the curvature of $\partial B$ show that the contribution of $\left|A_{B}\left(\rho^{-1}, \psi+\pi / 2\right)\right|$ is not larger than $c \rho^{-3 / 2}$ so that it suffices to consider $A_{B}\left(\rho^{-1}, \psi-\pi / 2\right)$ (which is a cap close to the origin).

We set more notation. For any $0 \leq \psi \leq \varepsilon$, we consider the straight line with slope $\psi$ and tangent to the curve $y=x^{\gamma}$ at a point $\left(x_{0}, x_{0}^{\gamma}\right)$. Then $A_{B}\left(\rho^{-1}, \psi-\pi / 2\right)$ is the set enclosed between the line $y=r(x)=$ 
$\gamma x_{0}^{\gamma-1}\left(x-x_{0}\right)+x_{0}^{\gamma}+(\rho \cos \psi)^{-1}$ and the curve $y=x^{\gamma}$. Let us call $x_{1}$ and $x_{2}$ the abscissae of the two points where they intersect (see Figure 4).

\section{Figure 4.}

Since $\tan \psi=\gamma x_{0}^{\gamma-1}$ we have

$$
c_{1} \psi \leq x_{0}^{\gamma-1} \leq c_{2} \psi
$$

We further split the interval $0 \leq \psi \leq \varepsilon$ into $0 \leq \psi \leq c \rho^{-1+1 / \gamma}$ and $c \rho^{-1+1 / \gamma} \leq \psi \leq \varepsilon$ for some suitable constant $c$.

Assume

$$
0 \leq \psi \leq c \rho^{-1+1 / \gamma}
$$

Since $\psi$ is positive, $\left|A_{B}\left(\rho^{-1}, \psi-\pi / 2\right)\right| \leq c \rho^{-1} x_{2}$. We recall that $x_{2}$ is the largest solution of the equation

$$
x^{\gamma}=\gamma x_{0}^{\gamma-1}\left(x-x_{0}\right)+x_{0}^{\gamma}+(\rho \cos \psi)^{-1}
$$

We now estimate $x_{2}$. This gives a bound for $\left|A_{B}\left(\rho^{-1}, \psi-\pi / 2\right)\right|$ since the assumption $\psi \geq 0$ yields $x_{2} \geq\left|x_{1}\right|$. To do this we observe that (3.14) implies that the above equation has no solutions for $x>k \rho^{-1 / \gamma}$ 
for $k$ sufficiently large. Indeed, (3.13) and (3.14) imply $x_{0} \leq c_{3} \rho^{-1 / \gamma}$ and therefore

$$
\begin{aligned}
x^{\gamma}-\gamma x_{0}^{\gamma-1}( & \left.x-x_{0}\right)-x_{0}^{\gamma}-(\rho \cos \psi)^{-1} \\
& >x^{\gamma}-c_{4} \rho^{-1+1 / \gamma} x-c_{4} \rho^{-1}-(\rho \cos \psi)^{-1} \\
& >\rho^{-1}\left(\left(\rho^{1 / \gamma} x\right)^{\gamma}-c_{4} \rho^{1 / \gamma} x-c_{4}-(\cos \psi)^{-1}\right) \\
& >0
\end{aligned}
$$

when $\rho^{1 / \gamma} x$ is larger than a suitable $k$. Then $x_{2} \leq k \rho^{-1 / \gamma}$ and

$$
\left|A_{B}\left(\rho^{-1}, \psi-\frac{\pi}{2}\right)\right| \leq c \rho^{-1-1 / \gamma}, \quad \text { for } 0 \leq \psi \leq c \rho^{-1+1 / \gamma} .
$$

Next, let $c \rho^{-1+1 / \gamma} \leq \psi \leq \varepsilon$. Then (3.13) and a suitable choice of the constant $c$ imply $x_{1}>0$. We want to show that

$$
x^{\gamma}-\gamma x_{0}^{\gamma-1}\left(x-x_{0}\right)-x_{0}^{\gamma}-(\rho \cos \psi)^{-1}
$$

becomes positive whenever $\left|x-x_{0}\right|>c_{5} \rho^{-1 / 2} x_{0}^{1-\gamma / 2}$. Towards this aim one checks the inequality

$$
(1+u)^{\gamma}-1-\gamma u \geq \frac{\gamma}{2} u^{2},
$$

which holds true for $\gamma>2$ and $u \geq-1$. Then

$$
\begin{aligned}
x^{\gamma}- & \gamma x_{0}^{\gamma-1}\left(x-x_{0}\right)-x_{0}^{\gamma}-(\rho \cos \psi)^{-1} \\
& =\left(x_{0}+\left(x-x_{0}\right)\right)^{\gamma}-\gamma x_{0}^{\gamma-1}\left(x-x_{0}\right)-x_{0}^{\gamma}-(\rho \cos \psi)^{-1} \\
& =x_{0}^{\gamma}\left(\left(1+\frac{x-x_{0}}{x_{0}}\right)^{\gamma}-\gamma \frac{x-x_{0}}{x_{0}}-1\right)-(\rho \cos \psi)^{-1} \\
& \geq x_{0}^{\gamma} \frac{\gamma}{2}\left(\frac{x-x_{0}}{x_{0}}\right)^{2}-(\rho \cos \psi)^{-1} \\
& \geq \frac{\gamma}{2} c_{5}^{2} \rho^{-1}-(\rho \cos \psi)^{-1} \\
& >0
\end{aligned}
$$

for a suitably large $c_{5}$. Consequently

$$
\left|x-x_{0}\right| \leq c_{5} \rho^{-1 / 2} x_{0}^{1-\gamma / 2},
$$


for any $x_{1} \leq x \leq x_{2}$. This and (3.13) show that

$$
\left|A_{B}\left(\rho^{-1}, \psi-\frac{\pi}{2}\right)\right| \leq c_{6} \rho^{-3 / 2} \psi^{(2-\gamma) /(2(\gamma-1))},
$$

for $c \rho^{-1+1 / \gamma} \leq \psi \leq \varepsilon$. Then (3.15), (3.18), the assumptions on the curvature of $\partial B$ and Lemma 3.8 yield

$$
\left|\widehat{\chi}_{B}(\rho \Theta)\right| \leq c_{7} \rho^{-1-1 / \gamma} .
$$

for any $\Theta$.

We now study the estimates of the $L^{p}$-norm, $2 \leq p<+\infty$. Because of the symmetry of $B$ it is enough to bound

$$
\begin{aligned}
& \left(\int_{-\pi / 2}^{\pi / 2}\left|\widehat{\chi}_{B}(\rho \Theta)\right|^{p} d \theta\right)^{1 / p} \leq\left(\int_{-\pi / 2}^{-\pi / 2+c \rho^{(1-\gamma) / \gamma}}\left|\widehat{\chi}_{B}(\rho \Theta)\right|^{p} d \theta\right)^{1 / p} \\
& +\left(\int_{-\pi / 2+c \rho^{(1-\gamma) / \gamma}}^{-\pi / 2+\varepsilon}\left|\widehat{\chi}_{B}(\rho \Theta)\right|^{p} d \theta\right)^{1 / p} \\
& +\left(\int_{-\pi / 2+\varepsilon}^{\pi / 2}\left|\widehat{\chi}_{B}(\rho \Theta)\right|^{p} d \theta\right)^{1 / p} \\
& =I_{1}+I_{2}+I_{3} \text {. }
\end{aligned}
$$

By the assumptions on the curvature of $\partial B$ we have $I_{3} \leq c_{8} \rho^{-3 / 2}$. Furthermore, by (3.19),

$$
I_{1} \leq c_{7} \rho^{-1-1 / \gamma}\left(\int_{0}^{c \rho^{(1-\gamma) / \gamma}} d \psi\right)^{1 / p} \leq c_{9} \rho^{-1-1 / p-1 / \gamma+1 /(\gamma p)} .
$$

In order to estimate $I_{2}$ we observe that Lemma 3.8, (3.18), the assumptions on the curvature of $\partial B$ and the choice $\gamma>2$ give

$$
\begin{aligned}
I_{2} \leq & c_{10} \rho^{-3 / 2}\left(\int_{c \rho^{(1-\gamma) / \gamma}}^{\varepsilon} \psi^{p(2-\gamma) /(2 \gamma-2)} d \psi\right)^{1 / p} \\
& \leq \begin{cases}c_{11} \rho^{-3 / 2}, & \text { for } p<\frac{2 \gamma-2}{\gamma-2} \\
c_{11} \rho^{-3 / 2}(\log \rho)^{(\gamma-2) /(2 \gamma-2)}, & \text { for } p=\frac{2 \gamma-2}{\gamma-2} \\
c_{11} \rho^{-1-1 / p-1 / \gamma+1 /(\gamma p)}, & \text { for } p>\frac{2 \gamma-2}{\gamma-2}\end{cases}
\end{aligned}
$$


In particular, for $p>(2 \gamma-2) /(\gamma-2)$,

$$
\left(\int_{0}^{2 \pi}\left|\widehat{\chi}_{B}(\rho \Theta)\right|^{p} d \theta\right)^{1 / p} \leq c_{12} \rho^{-1-1 / p-1 / \gamma+1 /(\gamma p)} .
$$

Observe that (3.20) cannot be obtained interpolating between $L^{2}$ and $L^{\infty}$. Moreover, we shall see in a moment that the above estimates are sharp and therefore

$$
\begin{aligned}
& \left\|\widehat{\chi}_{B}(\rho \cdot)\right\|_{L^{p}\left(\Sigma_{1}\right)} \\
& \approx \begin{cases}\rho^{-3 / 2}, & \text { for } p<\frac{2 \gamma-2}{\gamma-2}, \\
\rho^{-3 / 2}(\log \rho)^{(\gamma-2) /(2 \gamma-2)}, & \text { for } p=\frac{2 \gamma-2}{\gamma-2}, \\
\rho^{-1-1 / p-1 / \gamma+1 /(\gamma p)}, & \text { for } p>\frac{2 \gamma-2}{\gamma-2} .\end{cases}
\end{aligned}
$$

When $p<(2 \gamma-2) /(\gamma-2)$ the estimate from below follows from Lemma 3.6. We shall now prove the estimates from below in (3.21) when $p \geq$ $(2 \gamma-2) /(\gamma-2)$. Indeed, (3.17) can be reversed so that, by (3.13), $x \notin\left(x_{1}, x_{2}\right)$ implies

$$
\left|x-x_{0}\right| \geq c_{13} \rho^{-1 / 2} x_{0}^{1-\gamma / 2}
$$

whence

$$
\left|A_{B}\left(\frac{1}{\rho}, \psi-\frac{\pi}{2}\right)\right| \geq c_{13} \rho^{-3 / 2} \psi^{(2-\gamma) /(2(\gamma-1))},
$$

for $c \rho^{-1+1 / \gamma} \leq \psi \leq \varepsilon$. To prove this, we argue as for the estimate from above, after substituting (3.16) with the inequality

$$
(1+u)^{\gamma}-1-\gamma u \leq \gamma^{2} 2^{\gamma} u^{2}
$$

valid for $\gamma>2$ and $-1 \leq u \leq 1$. The restriction $u \leq 1$ causes no troubles since the monotonicity of the curvature of $y=x^{\gamma}$ implies $x_{2}-x_{0} \leq x_{0}-x_{1}$ for $c \rho^{-1+1 / \gamma} \leq \psi \leq \varepsilon$, so that

$$
-1 \leq \frac{x-x_{0}}{x_{0}} \leq 1
$$

if $x_{1} \leq x \leq x_{2}$. 
To estimate $\widehat{\chi}_{B}$, let $\psi$ be fixed in $c \rho^{-1+1 / \gamma} \leq \psi \leq \varepsilon$ and recall that $\theta=\psi-\pi / 2$. By Lemma 3.8 the decay of $\widehat{\chi}_{B}(\rho \Theta)$ depends on the shape of $\partial B$ at the point $\left(x_{0}, x_{0}^{\alpha}\right)$, see Figure 4 , and at the "opposite" point. The latter will turn out to give a negligible contribution because of our assumption on the curvature of $\partial B$ outside the origin. Then, if $\zeta \in C_{0}^{\infty}\left(\mathbb{R}^{2}\right), \zeta(x)=1$ in a neighborhood of the point $\left(x_{0}, x_{0}^{\alpha}\right)$, Lemma 3.14 allows us to apply Lemma 3.15 so to obtain

$$
\begin{aligned}
&\left(\int_{0}^{2 \pi}\left|\widehat{\chi}_{B}(\rho \Theta)\right|^{p} d \theta\right)^{1 / p} \\
& \geq\left(\int_{c \rho^{-1+1 / \gamma}}^{\varepsilon}\left|\widehat{\chi}_{B}(\rho \Theta)\right|^{p} d \psi\right)^{1 / p} \\
& \geq\left(\int_{c \rho^{-1+1 / \gamma}}^{\varepsilon}\left|\left[\zeta \chi_{B}\right]^{\wedge}(\rho \Theta)\right|^{p} d \psi\right)^{1 / p} \\
&-\left(\int_{c \rho^{-1+1 / \gamma}}^{\varepsilon}\left|\left[(1-\zeta) \chi_{B}\right]^{\wedge}(\rho \Theta)\right|^{p} d \psi\right)^{1 / p} \\
& \geq c_{14}\left(\int_{c \rho^{-1+1 / \gamma}}^{\varepsilon}\left|A_{B}\left(\frac{1}{\rho}, \psi-\frac{\pi}{2}\right)\right|^{p} d \psi\right)^{1 / p}-c_{15} \rho^{-3 / 2} \\
& \geq c_{16} \rho^{-1-1 / p-1 / \gamma+1 /(\gamma p)} .
\end{aligned}
$$

We recall that the above holds whenever $p>(2 \gamma-2) /(\gamma-2)$. This ends the proof once we observe that when $p>(2 \gamma-2) /(\gamma-2)$ we have $(2 p-2) /(p-2)<\gamma<\infty$ and therefore the range of the exponent

$$
1+\frac{1}{p}+\frac{1}{\gamma}-\frac{1}{\gamma p}
$$

is the open interval $(1+1 / p, 3 / 2)$.

The proof of the previous lemma can be used to get a result for singular measures supported on the curve $y=|x|^{\gamma}, \gamma>2$.

Proposition 3.17. Let $d \sigma$ be the measure on the curve $y=|x|^{\gamma}, \gamma>2$, induced by the Lebesgue measure on $\mathbb{R}^{2}$. Let $\kappa \in C_{0}^{\infty}\left(\mathbb{R}^{2}\right), \kappa(t)=1$ in a neighborhood of the origin and let $d \mu=\kappa(t) d \sigma$. Let $\ell\left(\rho^{-1}, \theta\right)$ be the 
length of the chord as in Figure 4 . Let $1 \leq p \leq \infty$, then

$$
\begin{aligned}
\left\|\ell\left(\rho^{-1}, \cdot\right)\right\|_{L^{p}([0,2 \pi))} & \approx\|\widehat{d \mu}(\rho \cdot)\|_{L^{p}\left(\Sigma_{1}\right)} \\
& \approx \begin{cases}\rho^{-1 / 2}, & \text { for } p<\frac{2 \gamma-2}{\gamma-2} \\
\rho^{-1 / 2}(\log \rho)^{(\gamma-2) /(2 \gamma-2)}, & \text { for } p=\frac{2 \gamma-2}{\gamma-2} \\
\rho^{-1 / p-1 / \gamma+1 /(\gamma p)}, & \text { for } p>\frac{2 \gamma-2}{\gamma-2}\end{cases}
\end{aligned}
$$

\section{A remark on the average decays associated to arbitrary convex sets.}

Let $\mathcal{C}$ be the space of convex bodies in $\mathbb{R}^{2}$ endowed with the Hausdorff metric $\delta^{H}$ defined by

$$
\delta^{H}(C, D)=\max \left\{\sup _{x \in C} \inf _{y \in D}|x-y|, \sup _{y \in D} \inf _{x \in C}|x-y|\right\},
$$

for $C, D \in \mathcal{C}$. A weak version of Blaschke selection theorem (see [9]) shows that $\left(\mathcal{C}, \delta^{H}\right)$ is locally compact and therefore of second category (not meager) by Baire theorem. We fix $n \in \mathbb{N}$. On $\mathcal{C}$ we consider the functional

$$
\Phi_{n}(B)=\left(\int_{0}^{2 \pi}\left|\widehat{\chi}_{B}(n \Theta)\right|^{p} d \theta\right)^{1 / p}
$$

and we observe that

$$
\begin{aligned}
\left|\left\|\widehat{\chi}_{C}(n \cdot)\right\|_{L^{p}\left(\Sigma_{1}\right)}-\left\|\widehat{\chi}_{D}(n \cdot)\right\|_{L^{p}\left(\Sigma_{1}\right)}\right| & \leq\left\|\widehat{\chi}_{C}(n \cdot)-\widehat{\chi}_{D}(n \cdot)\right\|_{L^{p}\left(\Sigma_{1}\right)} \\
& \leq|C \Delta D|,
\end{aligned}
$$

implies continuity of $\Phi_{n}$.

Next, let $1<p<2$ and $3 / 2<\gamma<1+1 / p$. Let $B$ be a convex set with piecewise smooth boundary. The results in the previous section show that the family $\left\{\Phi_{n}\right\}$ satisfies

$$
\Phi_{n}(B)=o\left(n^{-\gamma}\right)
$$

when $B$ is a polygon and

$$
n^{-\gamma}=o\left(\Phi_{n}(B)\right)
$$


if $B$ is not a polygon nor a cut disc. Therefore, the sets

$$
\mathcal{A}_{1}=\left\{B \in \mathcal{C}: \Phi_{n}(B)=o\left(n^{-\gamma}\right)\right\}
$$

and

$$
\mathcal{A}_{2}=\left\{B \in \mathcal{C}: n^{-\gamma}=o\left(\Phi_{n}(B)\right)\right\}
$$

are dense in $\mathcal{C}$. A similar argument also applies when $p>2$.

We now use the following result due to Gruber, [6].

Lemma 4.1. Let $T$ be a second category topological space.

i) Let $\alpha_{1}, \alpha_{2}, \cdots \in \mathbb{R}^{+}$and let $\phi_{1}, \phi_{2}, \cdots: T \longrightarrow \mathbb{R}^{+}$be continuous functions such that

$$
\mathcal{A}=\left\{x \in T: \phi_{n}(x)=o\left(\alpha_{n}\right) \text { as } n \longrightarrow+\infty\right\}
$$

is dense in T. Then for all, but a meager subset of $x$ 's belonging to $T$, the inequality $\phi_{n}(x)<\alpha_{n}$ holds for infinitely many $n$.

ii) Let $\beta_{1}, \beta_{2}, \cdots \in \mathbb{R}^{+}$and let $\psi_{1}, \psi_{2}, \cdots: T \longrightarrow \mathbb{R}^{+}$be continuous functions such that

$$
\mathcal{B}=\left\{x \in T: \beta_{n}=o\left(\psi_{n}(x)\right) \text { as } n \longrightarrow+\infty\right\}
$$

is dense in T. Then for all, but a meager subset of $x$ 's belonging to $T$, the inequality $\beta_{n}<\psi_{n}(x)$ holds for infinitely many $n$.

By way of summary we have.

Proposition 4.2. Let $1<p<2$ and $3 / 2<\gamma_{1} \leq \gamma_{2}<1+1 / p$ or let $2<p<\infty$ and $1+1 / p<\gamma_{1} \leq \gamma_{2}<3 / 2$. Then there exists a meager set $\mathcal{E} \subset \mathcal{C}$ such that for all $B \in \mathcal{C} \backslash \mathcal{E}$ there exist two sequences $n_{k}, m_{k}$ satisfying

$$
\left(\int_{0}^{2 \pi}\left|\widehat{\chi}_{B}\left(n_{k} \Theta\right)\right|^{p} d \theta\right)^{1 / p} \geq n_{k}^{-\gamma_{1}}
$$

and

$$
\left(\int_{0}^{2 \pi}\left|\widehat{\chi}_{B}\left(m_{k} \Theta\right)\right|^{p} d \theta\right)^{1 / p} \leq m_{k}^{-\gamma_{2}}
$$




\section{A result on the geometry of convex sets.}

At this point, little effort is needed to prove the following result, which may be of independent interest.

Theorem 5.1. Let $A_{B}(\delta, \theta)$ be as in (1.6) and let $\delta \leq 1 / 2$.

If $B$ is a polygon, then

$$
c_{1} \delta^{2} \log \left(\frac{1}{\delta}\right) \leq \int_{0}^{2 \pi} A_{B}(\delta, \theta) d \theta \leq c_{2} \delta^{2} \log \left(\frac{1}{\delta}\right),
$$

while if $B$ is not a polygon

$$
c_{1} \delta^{3 / 2} \leq \int_{0}^{2 \pi} A_{B}(\delta, \theta) d \theta \leq c_{2} \delta^{3 / 2} .
$$

Let $1<p \leq \infty$. Then the following are equivalent.

i) There exist $a>0$ and a convex body $B$ with $C^{2}$ boundary such that

$$
c_{1} \delta^{a} \leq\left(\int_{0}^{2 \pi} A_{B}(\delta, \theta)^{p} d \theta\right)^{1 / p} \leq c_{2} \delta^{a} .
$$

ii) The pair $(1 / p, a)$ belongs to the set $S \cup T$, where

$$
\begin{gathered}
S=\left\{\left(\frac{1}{p}, a\right): 1<p<2, a=\frac{3}{2} \text { or } a=1+\frac{1}{p}\right\}, \\
T=\left\{\left(\frac{1}{p}, a\right): 2 \leq p \leq \infty, 1+\frac{1}{p} \leq a \leq \frac{3}{2}\right\} .
\end{gathered}
$$

The proof of this theorem is largely a consequence of results in the previous section. Actually, the present problem is simpler since $A_{B}(\delta, \theta)$ is positive and no cancellation can arise. We sketch the argument for a reader specifically interested in this result.

Proof. We split the proof into several steps. We assume $\delta>0$ sufficiently small.

Step 1. Upper bound when $1 \leq p \leq 2$

$$
\left(\int_{0}^{2 \pi} A_{B}(\delta, \theta)^{2} d \theta\right)^{1 / 2} \leq c \delta^{3 / 2}
$$


for any $B$.

This has been proved by Podkorytov in [13, p. 60].

Step 2. If $P$ is a polygon, then

$$
\begin{gathered}
c_{1} \delta^{2} \log \left(\frac{1}{\delta}\right) \leq \int_{0}^{2 \pi} A_{P}(\delta, \theta) d \theta \leq c_{2} \delta^{2} \log \left(\frac{1}{\delta}\right) \\
c_{1} \delta^{1+1 / p} \leq\left(\int_{0}^{2 \pi} A_{P}(\delta, \theta)^{p} d \theta\right)^{1 / p} \leq c_{2} \delta^{1+1 / p}, \quad \text { for } 1<p \leq \infty
\end{gathered}
$$

These estimates are easy consequences of the argument in Lemma 3.9.

Step 3. Upper bound when $2 \leq p \leq \infty$

$$
\left(\int_{0}^{2 \pi} A_{B}(\delta, \theta)^{p} d \theta\right)^{1 / p} \leq c_{2} \delta^{1+1 / p},
$$

for any $B$.

The case $p=\infty$ is obvious; the case $2<p<\infty$ follows as in Lemma 3.13.

Step 4. Admissible decays when $2 \leq p \leq \infty$.

For any $2 \leq p \leq \infty$ and any $1+1 / p \leq a \leq 3 / 2$ there exists $B$ such that

$$
c_{1} \delta^{a} \leq\left(\int_{0}^{2 \pi} A_{P}(\delta, \theta)^{p} d \theta\right)^{1 / p} \leq c_{2} \delta^{a}
$$

This is precisely the content of Lemma 3.16.

Step 5. Lower bound for $1 \leq p \leq \infty$ when $B$ is not a polygon

$$
\left(\int_{0}^{2 \pi} A_{B}(\delta, \theta)^{p} d \theta\right)^{1 / p} \geq c \delta^{3 / 2}
$$

Indeed, if $B$ is not a polygon, there exists a regular arc in $\partial B$ which does not coincide with its chord. Then, at any point in this arc one can apply the following elementary observation. Let $f \in C^{2}[-1,1]$ be a real function satisfying $f(0)=f^{\prime}(0)=0$ and $0 \leq f^{\prime \prime}(x) \leq 2 c$ for any $x \in[-1,1]$. Writing $f(x)=\delta$ for $x=x_{1}$ and $x=x_{2}$, we have $\left|x_{1}-x_{2}\right| \geq 2 \sqrt{\delta / c}$. 


\section{Lattice points in large convex planar sets.}

From the Introduction we recall the following:

Definition 6.1. Let $\sigma \in S O(2)$ and $t \in \mathbb{T}^{2}$. The discrepancy function $D_{B}(\rho, \theta, t)$ is defined by

$$
\begin{aligned}
D_{B}(\rho, \sigma, t) & =\operatorname{card}\left(\left(\rho \sigma^{-1}(B)-t\right) \cap \mathbb{Z}^{2}\right)-\rho^{2}|B| \\
& =\sum_{m \in \mathbb{Z}^{2}} \chi_{\rho \sigma^{-1}(B)-t}(m)-\rho^{2}|B| .
\end{aligned}
$$

We prove the following result.

Theorem 6.2. Assume $B$ is a convex body in $\mathbb{R}^{2}$ with piecewise $C^{\infty}$ boundary, which is not a polygon. Let $1 \leq p \leq 2$, then

$$
c_{1} \rho^{1 / 2} \leq\left\|D_{B}(\rho, \cdot, \cdot)\right\|_{L^{p}\left(S O(2) \times \mathbb{T}^{2}\right)} \leq c_{2} \rho^{1 / 2} .
$$

Proof. The estimate from above is easy (and essentially known). Indeed a computation gives

$$
D_{B}(\rho, \sigma, \cdot)^{\wedge}(m)=\rho^{2} \widehat{\chi}_{B}(\rho \sigma(m)),
$$

for any $m \in \mathbb{Z}^{2}, m \neq 0$ (please note that the hat symbol in the left hand side and in the right hand side refer to the Fourier transform on $\mathbb{T}^{2}$ and on $\mathbb{R}^{2}$ respectively). Hence, by Lemma 3.1,

$$
\begin{aligned}
\int_{S O(2)} \int_{T^{2}}\left|D_{B}(\rho, \sigma, t)\right|^{2} d t d \sigma & =\rho^{4} \int_{S O(2)} \sum_{m \neq 0}\left|\widehat{\chi}_{B}(\rho \sigma(m))\right|^{2} d \sigma \\
& =\rho^{4} \sum_{m \neq 0} \int_{S O(2)}\left|\widehat{\chi}_{B}(\rho \sigma(m))\right|^{2} d \sigma \\
& \leq \rho^{4} \sum_{m \neq 0}|\rho m|^{-3} \\
& =c \rho .
\end{aligned}
$$

Therefore, whenever $1 \leq p \leq 2$,

$$
\left\|D_{B}(\rho, \cdot, \cdot)\right\|_{L^{p}\left(S O(2) \times \mathbb{T}^{2}\right)} \leq\left\|D_{B}(\rho, \cdot, \cdot)\right\|_{L^{2}\left(S O(2) \times \mathbb{T}^{2}\right)} \leq c_{2} \rho^{1 / 2} .
$$


On the other hand, for any $m \in \mathbb{Z}^{2}, m \neq 0$,

$$
\begin{aligned}
\left\|D_{B}(\rho, \cdot, \cdot)\right\|_{L^{p}\left(S O(2) \times \mathbb{T}^{2}\right)} & \geq\left\|D_{B}(\rho, \cdot, \cdot)\right\|_{L^{1}\left(S O(2) \times \mathbb{T}^{2}\right)} \\
& =\int_{S O(2)} \int_{\mathbb{T}^{2}}\left|D_{B}(\rho, \sigma, t)\right| d t d \sigma \\
& \geq \int_{S O(2)}\left|D_{B}(\rho, \sigma, \cdot)^{\wedge}(m)\right| d \sigma \\
& =\rho^{2} \int_{S O(2)}\left|\widehat{\chi}_{B}(\rho \sigma(m))\right| d \sigma
\end{aligned}
$$

We split the argument for the estimate from below into three cases.

First case. Suppose $B$ is not a cut disc (see Definition 3.3). Then, making use of Lemma 3.6, (6.1) implies

$$
\left\|D_{B}(\rho, \cdot, \cdot)\right\|_{L^{p}\left(S O(2) \times \mathbb{T}^{2}\right)} \geq c_{1} \rho^{1 / 2}
$$

Second case. Suppose we have a disc D. First assume

$$
\min _{n \in \mathbb{Z}}\left|2 \rho-\frac{1}{4}-n\right| \geq \frac{1}{10}
$$

Let $m=(1,0)$, then, by $(6.1)$, and the asymptotic of Bessel functions,

$$
\begin{aligned}
\left\|D_{D}(\rho, \cdot, \cdot)\right\|_{L^{p}\left(S O(2) \times \mathbb{T}^{2}\right)} & \geq \rho J_{1}(2 \pi \rho) \\
& =\pi^{-1} \rho^{1 / 2} \cos \left(2 \pi \rho-\frac{3}{4} \pi\right)+\mathcal{O}(1) \\
& \geq c \rho^{1 / 2} .
\end{aligned}
$$

On the other hand, when

$$
\min _{n \in \mathbb{Z}}\left|2 \rho-\frac{1}{4}-n\right| \leq \frac{1}{10}
$$

we choose $m=(2,0)$, then

$$
\left\|D_{D}(\rho, \cdot, \cdot)\right\|_{L^{p}\left(S O(2) \times \mathbb{T}^{2}\right)} \geq \pi^{-1} \rho^{1 / 2} \cos \left(4 \pi \rho-\frac{3}{4} \pi\right)+\mathcal{O}(1) \geq c \rho^{1 / 2} .
$$


Third case. Suppose $B$ is a cut disc, coming from a given disc $D$. Without loss of generality we can assume

$$
\{(\cos \theta, \sin \theta):|\theta| \leq \alpha \text { or }|\pi-\theta| \leq \alpha\} \subset \partial B,
$$

for a small $\alpha>0$. Let

$$
U=\left\{\left(\begin{array}{cc}
\cos \theta & \sin \theta \\
-\sin \theta & \cos \theta
\end{array}\right):|\theta|<\frac{\alpha}{2}\right\} .
$$

Then, for $m=(1,0)$ or $m=(2,0)$,

$$
\begin{aligned}
\| D_{B}(\rho, \cdot, \cdot) & \|_{L^{p}\left(S O(2) \times \mathbb{T}^{2}\right)} \\
& \geq \rho^{2} \int_{U}\left|\widehat{\chi}_{B}(\rho \sigma(m))\right| d \sigma \\
& \geq\left|\rho^{2} \int_{U}\right| \widehat{\chi}_{D}(\rho \sigma(m))\left|d \sigma-\rho^{2} \int_{U}\right| \widehat{\chi}_{D \backslash B}(\rho \sigma(m))|d \sigma| .
\end{aligned}
$$

Now the third case is a consequence of the second one if we prove that

$$
\int_{U}\left|\widehat{\chi}_{D \backslash B}(\rho \sigma(m))\right| d \sigma \leq c \rho^{-2} .
$$

Indeed $D \backslash B$ looks like in the following picture and therefore, by applying Lemma 3.8 to each one of the connected components of $D \backslash B$, we get

$$
\left|\widehat{\chi}_{D \backslash B}(\rho \sigma(m))\right| \leq c \rho^{-2}
$$

uniformly in $\sigma \in U$. 
Acknowledgements. We wish to thank S. Campi and C. Schütt for some interesting comments. We are grateful to R. Schneider for suggesting us the argument in Section 4. Finally we wish to thank L. Colzani for several suggestions he gave us during the preparation of the paper.

\section{References.}

[1] Brandolini, L., Fourier transform of characteristic functions and Lebesgue constants for multiple Fourier series. Colloq. Math. 65 (1993), 51-59.

[2] Brandolini, L., Colzani, L., A convergence theorem for multiple Fourier series. Unpublished.

[3] Brandolini, L., Colzani, L., Travaglini, G., Average decay of Fourier transforms and integer points in polyhedra. Arkiv. Math. 35 (1997), 253-275.

[4] Brandolini, L., Travaglini, G., Pointwise convergence of Fejer type means. Tohoku Math. J. 49 (1997), 323-336.

[5] Bruna, J., Nagel, A., Wainger, S., Convex hypersurfaces and Fourier transforms. Ann. of Math. 127 (1988), 333-365.

[6] Gruber, P., In most cases approximation is irregular. Rend. Sem. Mat. Univ. Politec. Torino 41 (1983), 20-33.

[7] Hardy, G., On Dirichlet's divisor problem. Proc. London Math. Soc. 15 (1916), 1-25.

[8] Huxley, M. N., Area, lattice points, and exponential sums. Oxford Science Publications, 1996.

[9] Kelly, P. J., Weiss, M. L., Geometry and convexity. J. Wiley-Interscience, 1979.

[10] Kendall, D. G., On the number of lattice points in a random oval. Quart. J. Math. Oxford Ser. 19 (1948), 1-26.

[11] Krätzel, E., Lattice points. Kluwer Academic Publisher, 1988.

[12] Montgomery, H. L., Ten lessons on the interface between analytic number theory and harmonic analysis. CBMS Regional Conference Series in Mathematics. 84, American Mathematical Society, 1994.

[13] Podkorytov, A. N., The asymptotic of a Fourier transform on a convex curve. Vestn. Leningr. Univ. Mat. 24 (1991), 57-65.

[14] Randol, B., On the Fourier transform of the indicator function of a planar set. Trans. Amer. Math. Soc. 139 (1969), 279-285.

[15] Randol, B., On the asymptotic behaviour of the Fourier transform of 
the indicator function of a convex set. Trans. Amer. Math. Soc. 139 (1969), 271-278.

[16] Ricci, F., Travaglini, G., In preparation.

[17] Schütt, C., The convex floating body and polyhedral approximation. Israel J. Math. 73 (1991), 65-77.

[18] Stein, E. M., Harmonic Analysis: real variable methods, ortogonality and oscillatory integrals. Princeton University Press, 1993.

[19] Varchenko, A. N., Number of lattice points in families of homothetic domains in $\mathbb{R}^{n}$. Funk. An. 17 (1983), 1-6.

Recibido: 28 de enero de 1.997

Revisado: 30 de octubre de 1.997

Luca Brandolini, Marco Rigoli and Giancarlo Travaglini Dipartimento di Matematica

Università di Milano Via Saldini 50 20133 Milano, ITALY brandolini@mat.unimi.it rigoli@mat.unimi.it travaglini@mat.unimi.it 